\title{
Fluctuations of One Dimensional Ginzburg-Landau Models in Nonequilibrium
}

\author{
Chih-Chung Chang ${ }^{1}$ and Horng-Tzer Yau ${ }^{2 \star, \star \star}$ \\ ${ }^{1}$ Institute of Applied Mathematics, National Tsing Hua University, Hsinchu 300, Taiwan, R.O.C. \\ ${ }^{2}$ Courant Institute of Mathematical Sciences, New York University, 251 Mercer Street, New \\ York, NY 10012, USA
}

Received April 24, 1991; in revised form September 9, 1991

\begin{abstract}
We study the fluctuation of one dimensional Ginzburg-Landau models in nonequilibrium along the hydrodynamic (diffusion) limit. The hydrodynamic limit has been proved to be a nonlinear diffusion equation by Fritz, GuoPapanicolaou-Varadhan, etc. We proved that if the potential is uniformly convex then the fluctuation process is governed by an Ornstein-Uhlenbeck process whose drift term is obtained by formally linearizing the hydrodynamic equation.
\end{abstract}

\section{Introduction}

In this paper we study the nonequilibrium fluctuations for a system of charges or spins in a one dimensional periodic lattice. Our model is generally known as a time dependent Ginzburg-Landau model (TDGL) with a conservation law. The dynamics in this model are governed by a conservation law together with random noise which also conserve the total charge. The resulting process is gradient and reversible with respect to a family of time independent Ginzburg-Landau (Gibbs) measures (TIGL).

The large scale behavior of TDGL model has been a subject of much interest. In particular, one can study the limit of the charge density under the diffusive type scalings - the hydrodynamical scaling limit of the charge density. Under various assumptions and generalities it has been proved $[4,5,6,8]$ that the hydrodynamic limit of the charge density in TDGL is governed by a nonlinear diffusion equation - the macroscopic equation. The macroscopic equation has also been studied considerably before and properties such as uniqueness, existence and a priori bounds are well known [see 8 and references therein]. Thus, the hydrodynamic

\footnotetext{
* Work partially supported by the National Science Foundation under grant no. DMS 8806731 and DMS 9101196

$\star \star$ A.P. Sloan Foundation fellow and David and Lucile Packard Foundation Fellow
} 
behavior of the charge density is in some way understood. [For a review of hydrodynamical scaling limit see 3 or 11.]

On the other hand, the behaviors of the more interesting fluctuations are much less understood. The only known result concerning fluctuations of this model is a theorem proved by Zhu [15] (following an approach outlined by Spohn [19]) and by Chang [2] which states that the limiting behavior of the fluctuations in equilibrium is governed by a stationary Ornstein-Uhlenbeck process. Their result is strictly restricted to GL models without finite range interactions. In this case the invariant measures degenerate into product measures and correlations come entirely from the noise. It should be noted that the assumption of equilibrium is critical in either Zhu's or Chang's approach. To gain some feeling of this, let us consider the nature and basic problem of estimating the susceptibility $\left(\right.$ i.e. $\left.\sum_{j=1}^{N}\left\langle x_{1}, x_{j}\right\rangle\right)$ at time $t$ assuming that it is bounded at $t=0$. In equilibrium the susceptibility is always zero because the density of the system is always a product. On the contrary in nonequilibrium it seems hopeless to obtain any bound on susceptibility at later time $t$ since the time derivative of the susceptibility generates infinitely many new terms! It is widely believed that, in fact, one should try to avoid the problem of bounding susceptibility directly in nonequilibrium.

Our main result of this paper is that the limiting behavior of the fluctuations in nonequilibrium is governed by a nonstationary Ornstein-Uhlenbeck process. Similar results concerning nonequilibrium fluctuations but for different models were obtained before by Ferrari-Presutti-Vares [18] and by Dietrich [17]. The methods we employ here are completely different from those of $[2,10,15,17-19]$. We first need an approach avoiding the problem of bounding susceptibility directly. Apart from this we have to bound the error of the hydrodynamic limit which is trivially equal to zero in the equilibrium. We note that in all previous works $[4,5,6,8]$ this control of error terms is out of reach since they all depend on some sort of compactness arguments. For this purpose, we give another proof of the hydrodynamic limit with error estimates to the accuracy we need. A sketch of our approach and bounds on the hydrodynamic errors will be given later in Sect. 2 .

We now introduce the setup. Let $S$ be the unit circle represented as the interval $[0,1]$ with 0 and 1 identified. For each positive integer $N$, their are $N$ scaled periodic lattice points located at sites $i / N$ in $S, i=1,2, \ldots, N$. Let $x_{i}(t)$ represent the continuous charges or magnetizations at site $i / N$ at time $t$. The dynamics of the charge configurations is governed by the infinitesimal generator

$$
L_{N}=\frac{N^{2}}{2}\left\{\sum_{i=1}^{N}\left(\partial_{i}-\partial_{i+1}\right)^{2}-\left(\phi^{\prime}\left(x_{i}\right)-\phi^{\prime}\left(x_{i+1}\right)\right)\left(\partial_{i}-\partial_{i+1}\right)\right\},
$$

where $\phi \in C^{1}(\mathbb{R})$ is a potential satisfying $\int e^{-\phi(x)} d x=1$. The generator $L_{N}$ is formally symmetric with respect to the product measure

$$
\Phi_{N}(d x)=\prod_{i=1}^{N} \exp \left[-\phi\left(x_{i}\right)\right] d x_{i}
$$

and hence defines a reversible process with invariant measure $\Phi_{N}(d x)$. The system (1.1) is called the one dimensional Ginzburg-Landau model (without finite range interactions). 
The evolution of TDGL is determined via the infinitesimal generator $L_{N}$ as follows. Starting with initial density $f_{N}\left(0 ; x_{1}, \ldots, x_{N}\right) \Phi_{N}$, the system at time $t$ will have a density $f_{N}\left(t ; x_{1}, \ldots, x_{N}\right) \Phi_{N}$ with $f_{N}$ satisfying

$$
\frac{\partial}{\partial t} f_{N}(t)=L_{N} f_{N}(t), \quad f_{N}(t=0)=f_{N}(0) .
$$

Equivalently, one can describe the dynamics by the stochastic differential equations

$$
d x_{i}=\frac{1}{2} N^{2}\left(\phi^{\prime}\left(x_{i+1}\right)-2 \phi^{\prime}\left(x_{i}\right)+\phi^{\prime}\left(x_{i-1}\right)\right) d t+N\left(d \beta_{i}-d \beta_{i+1}\right),
$$

where $\beta_{i}$ are independent Brownian motions.

Define the empirical measure by

$$
\mu_{N}=\frac{1}{N} \sum x_{i} \delta_{i / N}
$$

We shall say that the empirical measure defined by a distribution $f(x) \Phi_{N}(d x)$ has the macroscopic density $m(\theta)$ if for any smooth function $J$ and any $\delta>0$,

$$
\begin{gathered}
\lim _{N \rightarrow \infty} \int_{A_{N, J, \delta}} f(x) \Phi_{N}(d x)=0, \\
A_{N, J, \delta}=\left\{x:\left|\frac{1}{N} \sum J\left(\frac{i}{N}\right) x_{i}-\int J(\theta) m(\theta) d \theta\right|>\delta\right\} .
\end{gathered}
$$

We need the concept of entropy to state the hydrodynamic theorem of GPV [6]. Let us denote the relative entropy of $f$ w.r.t. $\Phi_{N}$ by

$$
H_{N}(f) \equiv \int f \log f \Phi_{N}(d x) .
$$

The main result of GPV can be summarized as, roughly speaking, that if the system has a macroscopic density $m_{0}(\theta)$ at time $t=0$ then the system has a macroscopic density $m(t, \theta)$ at later time $t$ provided the initial entropy is bounded by a constant times $N$, i.e.

$$
H_{N}\left(f_{N}(0)\right) \leqq C N .
$$

Furthermore, the density $m(t, \theta)$ satisfies the nonlinear diffusion equation

$$
\frac{\partial m}{\partial t}=\frac{1}{2} h^{\prime}(m(t, \theta))_{\theta \theta},\left.\quad m(t, \theta)\right|_{t=0}=m_{0}(\theta) .
$$

Here the function $h$ is the free energy defined by

$$
\begin{aligned}
& h(y)=\sup _{\lambda}[\lambda y-\rho(\lambda)], \\
& \rho(\lambda)=\log M(\lambda)=\log \int e^{\lambda x-\phi(x)} d x .
\end{aligned}
$$

The results of GPV have been extended to GL with finite range interactions by Rezakhanlou [8]. The results of [6] have also been proved previously by Fritz $[4,5]$ under stronger assumptions.

We pause to remark that we have followed the convention of notations in GPV which differs considerably from the usual convention in physics literature. 
For example, we have used $x_{i}$ to represent the "field" at the point $\frac{i}{N}$ and $\phi\left(x_{i}\right)$ for the potential of the "field" $x_{i}$, while in physics one uses $\phi(x)$ as the "field" at $x$ and $V(\phi(x))$ as the potential.

Define the empirical fluctuation field by

$$
\zeta_{i}^{N}(t)=\sqrt{N} \xi_{i}^{N}(t)=\sqrt{N}\left(x_{i}(t)-m\left(t, \frac{i}{N}\right)\right),
$$

where $m(t, \theta)$ is the solution to (1.9). Define the Sobolev norm by

$$
\|\zeta\|_{-\alpha}^{2}=\sum_{p \in \mathbb{Z}}|\hat{\zeta}(p)|^{2}\left(1+p^{2}\right)^{-\alpha}
$$

where $\hat{\zeta}(p)$ is the Fourier coefficient of $\zeta$ defined by

$$
\hat{\zeta}(p)=\frac{1}{N} \sum_{j=1}^{N} \zeta_{j} e^{i p j / N} .
$$

When $\alpha=1$ we make the following convention by defining

$$
\|\zeta\|_{-1}^{2}=\frac{1}{N} \sum_{i=1}^{N} \zeta_{i}\left[\left(-N^{2} \Delta\right)^{-1} \zeta\right]_{i}
$$

where $\left(-N^{2} \Delta\right)^{-1}$ is the inverse of the operator $-N^{2} \Delta$ with the discrete Laplacian $\Delta$ defined by $(\Delta \zeta)_{i}=\zeta_{i+1}-2 \zeta_{i}+\zeta_{i-1}$. Certainly the definition $\left(1.13^{\prime}\right)$ makes sense only if $\sum_{i=1} \zeta_{i}=0$. It is equivalent to (1.13) as $N \rightarrow \infty$.

Our assumptions for the main results are the following:

(A1) The potential $\phi$ is strictly convex, i.e., there exist two positive constants $\gamma_{1}$ and $\gamma_{2}$ s.t.

$$
0<\gamma_{1}<\phi^{\prime \prime}(x)<\gamma_{2}<\infty, \quad \forall x \in \mathbb{R} .
$$

(A2) The relative entropy of the initial distribution satisfies the linear bound $H_{N}\left(f_{N}(0)\right) \leqq C N$.

(A3) The initial fluctuation satisfies the following bounds.

$$
\begin{aligned}
& \varlimsup_{N \rightarrow \infty} N^{-1 / 2} E_{\text {non }}^{N}\left[\left\|\tilde{\zeta}_{0}^{N}\right\|_{-1}^{2}\right]=0, \quad \tilde{\zeta}_{0}^{N} \equiv \zeta_{0}^{N}-\sqrt{N}(\bar{x}-\bar{m}), \\
& \varlimsup_{N \rightarrow \infty} N^{1 / 2} E_{\text {non }}^{N}\left[|\bar{x}-\bar{m}|^{2}\right]=0 .
\end{aligned}
$$

Here $\bar{x}=\frac{1}{N} \sum_{i=1}^{N} x_{i}$ and $\bar{m}=\frac{1}{N} \sum_{i=1}^{N} m\left(\frac{i}{N}\right)$.

(A4) The initial fluctuation has a weak limit in $M\left(H_{-\alpha}\right)$ for some $\alpha>\frac{9}{2}\left(M\left(H_{-\alpha}\right)\right.$ denotes the space of measures on $H_{-\alpha}$ with weak topology), i.e. there is a measure $P_{0}^{\infty}$ in $M\left(H_{-\alpha}\right)$ s.t.

$$
\int f(\zeta) d P_{0}^{N}(\zeta) \rightarrow \int f(\zeta) d P_{0}^{\infty}(\zeta)
$$

for all $f$ bounded uniformly continuous on $H_{\alpha}$. Here $P_{0}^{N}$ is the initial law given by $f_{N}(0) \Phi_{N}$. 
We use the symbol $E_{\text {non }}^{N}$ for the expectation w.r.t. the process with initial distribution $f^{n}(0) \Phi_{N}$. When restricted to $t=0, E_{\text {non }}^{N}$ is the same as $E^{P_{0}^{N}}$. If $f^{N}(0) \equiv 1$ we write $E_{e q}^{N}$.

Note that (A3) is a very weak technical assumption since one expects that $E_{\text {non }}^{N}\left[\left\|\widetilde{\zeta}_{0}^{N}\right\|_{-1}^{2}\right]<\infty$ and $E_{\text {non }}^{N}\left[|\bar{x}-\bar{m}|^{2}\right] \sim N^{-1}$ if the central limit theorem holds at $t=0$. The assumption (A2), as will become clear later, will not be used in full strength in most parts of this paper. The assumption (A3) is inevitable since one needs "something" to begin with. The real main restriction comes from the assumption (A1). We make this assumption for the purpose of using the logarithmic Sobolev inequality.

We may now state our main results. For simplicity we restrict the time to the interval $[0,1]$. Certainly they hold for any finite interval.

Theorem 1. Let $P^{N}$ denote the stochastic process governed by (1.2) or (1.3). Suppose that the assumptions (A1)-(A4) are satisfied. Then $P^{N}$ converges weakly in $C([0,1]$, $\left.H_{-\alpha}\right)(\alpha>9 / 2)$ to a generalized Ornstein-Uhlenbeck process characterized by the SDE

$$
d \zeta^{\infty}(t)=\frac{1}{2} \partial_{\theta} \partial_{\theta}\left(h^{\prime \prime}(m(t, \theta)) \zeta^{\infty}(t)\right) d t+d \partial_{\theta} B(t),
$$

where $\partial_{\theta} B(t)$ is the Gaussian random field with covariance

$$
E\left[\partial_{\theta} B(s)\left(J_{1}\right) \partial_{\theta} B(t)\left(J_{2}\right)\right]=\delta(s-t) \int J_{1}^{\prime}(\theta) J_{2}^{\prime}(\theta) d \theta .
$$

Theorem 2. Suppose that the potential $\phi$ satisfies the assumption (A1) and the initial distribution satisfies (A1)-(A3). Then we have

(1) The bounds in (A3) continuous to hold at any time $t>0$.

(2) Let l be a positive parameter other than $N$ and let $2 K+1=\sqrt{N}(2 l+1)$. Let $\bar{x}_{i, K}(t)$ 's denote the average of $x_{j}(t)$ 's with $|j-i| \leqq K$. Then

$$
\lim _{l \rightarrow \infty} \varlimsup_{N \rightarrow \infty} E_{\text {non }}^{N} \int_{0}^{t} \frac{1}{\sqrt{N}} \sum_{i=1}^{N}\left(\bar{x}_{i, K}(t)-m\left(s, \frac{i}{N}\right)\right)^{2} d s=0
$$

uniformly on compact t-intervals.

Remark 1. Theorem 2 indeed holds if one replaces (A2) by a weaker assumption $H\left(f_{N}(0)\right) \leqq o\left(N^{4 / 3}\right)$. Since this is not needed in proving Theorem 1, we choose (A2) for simplicity. Interested readers can check the proof and find the optimal assumptions.

Remark. 2. If one is only interested in proving "low of large numbers," one can choose $K$ to be independent of $N$, namely $K=l$. In this case, a form of Theorem 2 holds under much weaker assumptions. It holds for GL model with finite range interactions in any dimension with initial entropy of order less than $N^{d+2}$.

The rest of this paper is organized as follows. In Sect. 2 we give outlines of proofs of Theorems 1 and 2. The details appear in Sects. 3-5. Theorem 2 is proved in Sect. 4.

\section{Sketch of Proofs for Theorem 1 and Theorem 2}

Let us start with a sketch of proof of Theorem 1 assuming Theorem 2.

First we replace $m\left(t, \frac{i}{N}\right)$ in $\zeta_{i}$ by $m_{i}(t)$ which is the solution of the discrete 
equation

$$
\begin{aligned}
d m_{i}(t) & =\frac{N^{2}}{2}\left(h^{\prime}\left(m_{i+1}\right)-2 h^{\prime}\left(m_{i}\right)+h^{\prime}\left(m_{i-1}\right)\right) d t=\frac{N^{2}}{2} \Delta\left(h^{\prime}\left(m_{i}\right)\right) d t \\
m_{i}(0) & =m\left(0, \frac{i}{N}\right) .
\end{aligned}
$$

One can check that $\left|m(i / N, t)-m_{i}(t)\right|<$ const. $N^{-1}$ [9]. Hence this replacement does not affect our results in this paper since we are interested up to order $N^{-1 / 2}$. From now on we shall use $m_{i}(t)$ instead of $m(i / N, t)$ without further explanation.

Note that we have abused notation of $\Delta$ in (2.1). A slightly clearer notation would write $\Delta\left(h^{\prime}\left(m_{i}\right)\right)$ as $\left[\Delta\left(h^{\prime}(m(\cdot))\right)\right]_{i}$. We shall however continue to use this simplified notation.

Combining (1.3) and (2.1),

$$
d \zeta_{i}=\frac{N^{5 / 2}}{2} \Delta\left(\phi^{\prime}\left(x_{i}\right)-h^{\prime}\left(m_{i}\right)\right) d t+N^{3 / 2}\left(d \beta_{i}-d \beta_{i+1}\right) .
$$

It is easy to see that the last term of (2.2) converges to the last term in (1.15). The main difficulty in proving Theorem 1 , as in the case of hydrodynamic limit, is to show that in a sense

$$
N^{5 / 2} \Delta\left(\phi^{\prime}\left(x_{i}\right)-h^{\prime}\left(m_{i}\right)\right) \Rightarrow \partial_{\theta} \partial_{\theta} h^{\prime \prime}(m(t, \theta)) \zeta^{\infty}(t) .
$$

We know from the proof of hydrodynamic limit in GPV that $\phi^{\prime}\left(x_{i}\right) \sim h^{\prime}\left(\bar{x}_{i, K}\right)$ with $\bar{x}_{i, K}$ defined in Theorem 2. For simplicity of notation, we omit the subscript $K$ in this section. Hence we decompose $\phi^{\prime}\left(x_{i}\right)-h^{\prime}\left(m_{i}\right)$ as

$$
N^{5 / 2} \Delta\left(\phi^{\prime}\left(x_{i}\right)-h^{\prime}\left(m_{i}\right)\right)=N^{5 / 2} \Delta\left[\left(\phi^{\prime}\left(x_{i}\right)-h^{\prime}\left(\bar{x}_{i}\right)\right)+h^{\prime}\left(\bar{x}_{i}\right)-h^{\prime}\left(m_{i}\right)\right] .
$$

The second term on the right side can be written by Taylor expansion as

$$
h^{\prime}\left(\bar{x}_{i}\right)-h^{\prime}\left(m_{i}\right)=h^{\prime \prime}\left(m_{i}\right)\left(\bar{x}_{i}-m_{i}\right)+O\left(\left(\bar{x}_{i}-m_{i}\right)^{2}\right) .
$$

It is not difficult to see that the first term $N^{5 / 2} \Delta h^{\prime \prime}\left(m_{i}\right)\left(\bar{x}_{i}-m_{i}\right)$ converges to $\partial_{\theta} \partial_{\theta} h^{\prime \prime}(m(t, \theta)) \zeta^{\infty}$. Hence our main job is to show that the error terms converges weakly to zero, i.e.,

$$
\begin{aligned}
N^{5 / 2} \Delta\left[\phi^{\prime}\left(x_{i}\right)-h^{\prime}\left(\bar{x}_{i}\right)\right] & \Rightarrow \delta_{0}, \\
N^{5 / 2} \Delta\left(\bar{x}_{i}-m_{i}\right)^{2} & \Rightarrow \delta_{0} .
\end{aligned}
$$

Equation (2.7) holds by Theorem 2 if $K$ is chosen as in Theorem 2. For (2.6) note that it requires in a sense that $\phi^{\prime}\left(x_{i}\right)-h^{\prime}\left(\bar{x}_{i}\right) \sim o\left(N^{-1 / 2}\right)$ since $N^{2} \Delta \sim \partial_{\theta} \partial_{\theta}$ is an operator of order 1 . Should one be interested only in the hydrodynamic limit, the required bound for $\phi^{\prime}\left(x_{i}\right)-h^{\prime}\left(\bar{x}_{i}\right)$ is much weaker, namely $\phi^{\prime}\left(x_{i}\right)-h^{\prime}\left(\bar{x}_{i}\right) \sim o(1)$. In general, we are unable to accomplish the bound of $o\left(N^{-1 / 2}\right)$. In the case in which $\phi$ is convex, we have logarithmic Sobolev inequality and a bound of $o\left(N^{-1 / 2}\right)$ is possible if one uses some large deviation estimates. We shall delay this until we sketch a proof of Theorem 1 since a similar estimate is needed there too. For now we only remark upon an important feature of (2.6).

Let us first pretend that the family $\left\{\phi^{\prime}\left(x_{i}(t)\right)-h^{\prime}\left(\bar{x}_{i}(t)\right)\right\}$ is a family of i.i.d. for each time $t$ fixed. Then (2.6) is clearly wrong since $N^{1 / 2}\left(\phi^{\prime}\left(x_{i}\right)-h^{\prime}\left(\bar{x}_{i}\right)\right)$ is order 1 
by central limit theorem. In our case $\left\{\phi^{\prime}\left(x_{i}\right)-h^{\prime}\left(\bar{x}_{i}\right)\right\}$ is certainly not i.i.d., but we cannot hope for anything better than space-time CLT since our goal is to prove a version of CLT, i.e. a generalized Ornstein-Uhlenbeck process. What saves us here is that (2.6) holds only after time average. In other words, even though $N^{1 / 2}\left(\phi^{\prime}\left(x_{i}\right)-h^{\prime}\left(\bar{x}_{i}\right)\right)$ is an object of order 1 for each time $t$ fixed, it fluctuates so much that it vanishes after time averaging. This cancellation effect of fluctuations is usually referred to as Boltzmann-Gibbs principle and is the hardest part of our proof. As we shall see later, our proof of " $\left(\phi^{\prime}\left(x_{i}\right)-h^{\prime}\left(\bar{x}_{i}\right)\right)=O\left(N^{-1 / 2}\right)$ " is considerably easier than the proof of Boltzmann-Gibbs principle.

We now sketch a proof of Theorem 2. From (2.4) it is easy to compute that

$$
N^{-1 / 2} d\|\tilde{\zeta}\|_{-1}^{2}=-N^{-1 / 2} \sum_{i} G_{i} d t+d M,
$$

where $d M$ is the martingale term and $G_{i}=F_{i}-T_{i}$ is defined by

$$
\begin{aligned}
T_{i} & =(\bar{x}-\bar{m})\left(\phi^{\prime}\left(x_{i}\right)-h^{\prime}\left(m_{i}\right)\right), \\
F_{i} & =\left(x_{i}-m_{i}\right)\left(\phi^{\prime}\left(x_{i}\right)-h^{\prime}\left(m_{i}\right)\right)-1 .
\end{aligned}
$$

If we assume a form of local ergodicity that $\Sigma g_{i}\left(x_{i}\right)$ can be replaced by $\Sigma \hat{g}_{i}\left(\bar{x}_{i}\right)$ with $\hat{g}_{i}$ defined by

$$
\hat{g}_{i}(y)=M(\lambda)^{-1} \int g_{i}(x) \exp (\lambda x-\phi(x)) d x, \quad \lambda=h^{\prime}(y) .
$$

Theorem 2 follows by taking expectation of (2.9) since

$$
\begin{aligned}
\hat{F}_{i}(y)-\hat{T}_{i}(y) & =\left(y-m_{i}\right)\left(h^{\prime}(y)-h^{\prime}\left(m_{i}\right)\right)-(\bar{x}-\bar{m})\left(h^{\prime}(y)-h^{\prime}\left(m_{i}\right)\right) \\
& \geqq \varepsilon\left(y-m_{i}\right)^{2}-2 \varepsilon^{-1}(\bar{x}-\bar{m})^{2} .
\end{aligned}
$$

Here the equality is just a simple computation while the Schwartz inequality and convexity of $h$ are used in the last inequality. Therefore, once one realizes the key relations (2.8)-(2.11), the proof of Theorem 2 depends on the control of $g\left(x_{i}\right)-\hat{g}\left(\bar{x}_{i}\right)$ which appears also in the proof of Theorem 1.

Let us denote by $X$ our key object to be bounded, namely,

$$
X=N^{-1 / 2} \int_{0}^{t}\left[\sum\left(g\left(x_{i}(s)\right)-\hat{g}\left(\bar{x}_{i}(s)\right)\right) J(i / N)\right] d s
$$

We shall bound $X$ by entropy method. Recall the entropy inequality asserting that for any two probability measures $\mu$ and $v$ one has

$$
\int h d v \leqq \beta^{-1} \log \int \exp (\beta h) d \mu+\beta^{-1} H(v / \mu), \quad \beta>0 .
$$

Hence the expectation of $X$ w.r.t. the nonequilibrium process starting with $f_{0}$ can be bounded by

$$
E_{\text {non }}^{N}(X) \leqq(\beta N)^{-1} \log E_{\mathrm{eq}}^{N}\left(e^{\beta N X}\right)+H_{N} / \beta N, \quad \beta>0 .
$$

As $\beta \rightarrow \infty$, the last term tends to zero by assumption $H_{N} \leqq C N$. The first term on the right side of (2.13) can be bounded using Feynman-Kac formula or the large deviation bound [14] by:

$$
\frac{t}{\beta N} \sup _{\int f^{2} \Phi_{N}=1}\left[\int \Phi_{n}(d x) \beta N^{1 / 2}\left\{\sum_{j}\left(g\left(x_{i}\right)-\hat{g}\left(\bar{x}_{i}\right)\right) J\left(\frac{i}{N}\right) f^{2}\left(x_{1} \cdots x_{N}\right)\right\}-N^{2} I_{N}(f)\right]
$$


where $I_{N}(f)$ is the Dirichlet form of $f$ defined by

$$
\begin{aligned}
I_{N}(f) & =\sum_{i=1}^{N} I_{i}(f) \\
I_{i}(f) & =\int\left(\frac{\partial f}{\partial x_{i}}-\frac{\partial f}{\partial x_{i+1}}\right)^{2} \Phi_{N}(d x) .
\end{aligned}
$$

We have now reduced everything to the $N$-body eigenvalue problem (2.14). This eigenvalue problem, although difficult, is tractable when the potential $\phi$ is convex and we have the logarithmic Sobolev inequality. The details to bound (2.14) will be given in the next section.

\section{Bounds on Principal Eigenvalue}

We shall prove in this section several estimates regarding the principal eigenvalue of operators of the form (2.14). As we have explained in Sect. II, they are our key technical estimates. The main results of this section will be summarized in Theorem 3.5. Our basic tools are logarithmic Sobolev inequality and asymptotic expansions of the central limit theorem. The logarithmic Sobolev inequality is in some sense a substitute of Rayleigh-Schrödinger perturbation theory. Our results can also be obtained by the Rayleigh-Schrödinger perturbation expansion.

Let us start with the following lemma concerning the asymptotic expansion of large deviation for i.i.d. Since it is a more or less known result, we shall delay its proof to the appendix.

Let $\Phi_{K}(d x)$ and $\Phi_{K, y}(d x)$ be the probability measure defined by

$$
\begin{gathered}
\Phi_{K}(d x)=\prod_{i=-K}^{K} \exp \left(-\phi\left(x_{j}\right)\right) d x_{j}, \\
\Phi_{K, y}(d x)=\delta(\bar{x}-y) \Phi_{K}(d x) Z_{K, y}^{-1}, \quad \bar{x}=(2 K+1)^{-1} \sum_{j=-K}^{K} x_{j},
\end{gathered}
$$

where $Z_{K, y}$ is the normalization factor.

Lemma 3.1. Suppose that $\phi$ satisfies the convexity bound (A1) and $F$ satisfies

$$
|F(x)| \leqq c_{0}\left(1+|x|^{2}\right)
$$

Then the following bound holds for

$$
\begin{gathered}
|\beta|<(2 K+1) \min \left(1, \gamma_{1} / 2 c_{0}\right) . \\
\beta^{-1} \log \int \exp \left[\beta(2 K+1)^{-1} \sum_{j=-K}^{K} F\left(x_{j}\right)\right] \Phi_{K, y}(d x) \\
=\langle F\rangle_{y}+G_{1}(F, y)(2 K+1)^{-1}+\beta G_{2}(F, y)(2 K+1)^{-1}+O\left(\left(1+\beta^{2}\right) / K^{2}\right), \\
G_{1}(F, y)=\frac{1}{2}\left[\langle x ; x ; x\rangle_{y}\langle x ; F\rangle_{y}\langle x ; x\rangle_{y}^{-2}-\langle x ; x ; F\rangle_{y}\langle x ; x\rangle_{y}^{-1}\right], \\
G_{2}(F, y)=\frac{1}{2}\left[\langle F ; F\rangle_{y}-\langle x ; F\rangle_{y}^{2}\langle x ; x\rangle_{y}^{-1}\right] .
\end{gathered}
$$


Here the expectation \langle\rangle$_{y}$ is defined by

$$
\langle f\rangle_{y}=M(\lambda)^{-1} \int f(x) \exp (\lambda x-\phi(x)) d x, \quad \lambda=h^{\prime}(y),
$$

where $h$ is defined in (1.11). As usual, $\langle;\rangle$ denotes the truncated (or corrected) correlation.

Corollary 3.2. Define $\hat{F}_{K}$ by

$$
\hat{F}_{K}(y)=\int(2 K+1)^{-1} \sum_{j=-K}^{K} F\left(x_{j}\right) \Phi_{K, y}(d x) .
$$

Then

$$
\hat{F}_{K}(y)=\langle F\rangle_{y}+G_{1}(F, y)(2 K+1)^{-1}+O\left(K^{-2}\right) .
$$

Proof of the Corollary. By convexity we have for all $\beta>0$,

$$
\begin{gathered}
-\beta^{-1} \log \int \exp \left[-\beta(2 K+1)^{-1} \sum_{j=-K}^{K} F\left(x_{j}\right)\right] \Phi_{K, y} d x \leqq \hat{F}_{K}(y) \\
\leqq \beta^{-1} \log \int \exp \left[\beta(2 K+1)^{-1} \sum_{j=-K}^{K} F\left(x_{j}\right)\right] \Phi_{K, y}(d x) .
\end{gathered}
$$

Choose $\beta=1 / K$ and we have Corollary 3.2.

Let $\left\{F^{(i)}\right\}$ be a family of functions indexed by $i, i=1, \ldots, N$. Suppose $K$ is a positive integer less than $N$. For $\tau>0$, define

$$
\begin{gathered}
V_{i}(F, K, \tau)=(2 K+1)^{-1} \sum_{|j-i| \leqq K}\left[F^{(i)}\left(x_{j}\right)-\hat{F}_{K}^{(i)}\left(\bar{x}_{i, K}\right)\right] 1\left(\left|\bar{x}_{i, K}\right|<\tau\right), \\
y_{i}=\bar{x}_{i, K}=(2 K+1)^{-1} \sum_{|j-i| \leqq K} x_{j}, \\
\langle J, V(F, K, \tau)\rangle=N^{-1} \sum_{i=1}^{N} J(i / N) V_{i}(F, K, \tau) .
\end{gathered}
$$

Lemma 3.3. Suppose that $\left\{F^{(i)}\right\}$ and $\phi$ satisfy (3.3) (uniformly in $i$ ) and (A1) respectively. Let $K<N$ be a positive integer and let $l=K N^{-1 / 2}$. Then for $\beta$ satisfying

$$
|\beta|\|J\|_{\infty}<\text { const. } N^{3 / 2} K^{-2}
$$

we have the following bound uniformly in $\tau$.

$$
\begin{aligned}
\sup \operatorname{spec}\left\{\beta N^{1 / 2}\langle J, V(F, K, \tau)\rangle-N I_{N}\right\} & \leqq \text { const. }\left[\alpha^{2} l^{2}+\alpha l^{-2} N^{-1 / 2}\right], \\
\alpha & \equiv|\beta|\|J\|_{\infty} .
\end{aligned}
$$

Here $I_{N}$ is the Dirichlet form defined in (2.15) and sup spec means sup spectrum. Furthermore, if $F^{(i)}$ satisfies the linear bound

$$
\left|F^{(i)}(x)\right| \leqq C(1+|x|)
$$

uniformly in $i$ then (3.17) holds for all $\beta$ and $J$ independent of assumption (3.16).

Proof. Denote $\sum_{|j-i| \leqq K} I_{j}=I_{i, K}$. Then the left side of (3.17) is bounded by

$$
\text { const. } \sup _{i} \sup _{|\alpha| \leqq \beta\|J\|_{\infty}} \sup \operatorname{spec} N^{1 / 2}\left[\alpha V_{i}(F, K, \tau)-l^{-3} K^{2} I_{i, K}\right] \text {. }
$$


Since our estimate will be the same for each $i$, we shall assume $i=0$ and drop the subscript (or superscript) $i$. We first observe that $I_{K}$ is infinite degenerate. But the degeneracy can be removed by keeping the average $\bar{x}=(2 K+1)^{-1} \sum_{|j| \leqq K} x_{j}$ fixed.

Since $V$ vanishes when $\bar{x}>\tau$, we only have to bound the sup spec for $\bar{x}$ lies inside the compact interval $|\bar{x}| \leqq \tau$. We shall assume for simplicity of notation that $\bar{x}=0$. Let us choose $y_{i}=x_{i}, i=-K, \ldots, K-1$ as independent variable in the hyperplane $\bar{x}=0$ and define

$$
\tilde{I}_{K}=\int \sum_{i=-K}^{K-1}\left(\frac{\partial f}{\partial y_{i}}\right)^{2} \Phi_{K, 0}(d x) .
$$

Clearly by change of variable and Schwartz inequality

$$
\begin{aligned}
\sum_{i=-K}^{K-1}\left(\frac{\partial f}{\partial y_{i}}\right)^{2} & =\sum_{i=-K}^{K-1}\left(\frac{\partial f}{\partial x_{i}}-\frac{\partial f}{\partial x_{K}}\right)^{2} \\
& \leqq \text { const. } K^{2} \sum_{i=-K}^{K-1}\left(\frac{\partial f}{\partial x_{i}}-\frac{\partial f}{\partial x_{i+1}}\right)^{2} .
\end{aligned}
$$

Also, one can check that the density $\Phi_{K, 0}$ is log concave (by assumption (A1)) and one has the logarithmic Sobolev inequality [16].

$$
\tilde{I}_{K}(t) \geqq \text { const. } \int f^{2} \log f^{2} \Phi_{K, 0}(d x)=\text { const. } H\left(f^{2}\right) .
$$

Combining (3.20) to (3.22), we can bound (3.19) by

$$
\text { const. } N^{1 / 2} l^{-3} \sup _{\int f^{2} \Phi_{K, 0}(d x)=1}\left\{\alpha l^{3} \int V(F, K, \tau) f^{2} \Phi_{K, 0}(d x)-H\left(f^{2}\right)\right\} \text {. }
$$

By entropy inequality (2.12) we can bound (3.23) by

$$
\text { const. } N^{1 / 2} l^{-3} \log \left\{\int \exp \left[\alpha l^{3} V(F, K, \tau)\right] \Phi_{K, 0}(d x)\right\} \text {. }
$$

By Lemma 3.1 and (3.11) we can bound (3.24) by

$$
\text { const. } \alpha N^{1 / 2}\left\{\alpha l^{3} K^{-1} G_{2}(F, 0)+\left(1+\alpha^{2} l^{6}\right) K^{-2}\right\},
$$

provided that

$$
\alpha l^{3} \leqq \text { const. } K=\text { const. } N^{1 / 2} l .
$$

Since $G_{2}(F, 0)$ is bounded, the first term in the bracket of (3.25) is bounded by const. $\alpha l^{2} N^{-1 / 2}$. Hence (3.25) is bounded by

$$
\text { const. }\left[\alpha^{2} l^{2}+\alpha l^{-2} N^{-1 / 2}+\alpha^{3} l^{4} N^{-1 / 2}\right] \leqq \text { const. }\left[\alpha^{2} l^{2}+\alpha l^{-2} N^{-1 / 2}\right],
$$

provided that the assumption (3.16) is satisfied. This proves (3.17). For the case $F$ satisfies (3.18) we only have to prove that (3.24) is bounded by (3.27) if (3.26) is violated, namely $\alpha>$ const. $N^{1 / 2} l^{-2}$. By the assumption (3.18) and Schwartz inequality

$$
\begin{aligned}
\alpha l^{3} V(F, K) & \leqq \text { const. } \alpha l^{3} K^{-1} \sum_{j=-K}^{K}\left(\left|x_{j}\right|+1\right) \\
& \leqq \text { const. }\left[\varepsilon^{-1} \alpha^{2} l^{6} K^{-1}+\varepsilon \sum x_{j}^{2}\right]
\end{aligned}
$$


Hence (3.24) is bounded by

$$
\text { const. }\left\{\varepsilon^{-1} \alpha^{2} l^{2}+N^{1 / 2} l^{-3} \log \int \exp \left[\varepsilon \sum x_{j}^{2}\right] \Phi_{K, 0}(d x)\right\} \text {. }
$$

By choosing $\varepsilon$ small enough, we can bound the integral by $\exp$ (const. $K$ ). Thus (3.29) is bounded by const. $\left(\alpha^{2} l^{2}+l^{-2} N\right) \leqq$ const. $\alpha^{2} l^{2}$, since $\alpha \geqq N^{1 / 2} l^{-2}$. Combining this with (3.19) $\sim(3.24)$, we have proved (3.17) independent of $(3.16)$ provided that (3.18) is satisfied.

Lemma 3.4. Let $L$ be a positive integer of order $N^{3 / 10}$ and let $M=(2 K+1) /(2 L+1)$ be an integer. Denote $\hat{F}_{L}^{(i)}(\cdot) 1(|\cdot|<\tau)=\hat{F}_{L, \tau}^{(i)}(\cdot)$ and define $V\left(F, K, L, \tau, \tau^{\prime}\right)$ by

$$
V_{i}\left(F, K, L, \tau, \tau^{\prime}\right)=M^{-1} \sum_{\alpha=1}^{M}\left[\hat{F}_{L, \tau}^{(i)}\left(y_{i \alpha}\right)-\hat{F}^{(i)}\left(y_{i}\right)\right] 1\left(\left|y_{i}\right|<\tau^{\prime}\right),
$$

where $y_{i}=\bar{x}_{i, K}$ and $y_{i \alpha}$ is the average of $x$ in the $\alpha^{\text {th }}$ box of $(2 L+1)$ sites around $i$, i.e.

$$
y_{i \alpha} \equiv(2 L+1)^{-1} \sum_{j \in \alpha(i)} x_{j} \equiv(2 L+1)^{-1} \sum_{j=i-K+(2 L+1)(\alpha-1)}^{i-K+(2 L+1) \alpha-1} x_{j} .
$$

If $\tau^{\prime}<\tau$ are large enough and $L \sim N^{3 / 10}$ then for any constant $\beta$ (independent of $N$ )

$$
\varlimsup_{l \rightarrow \infty} \varlimsup_{N \rightarrow \infty} \beta N^{1 / 2}\left\langle J, V\left(F, K, L, \tau, \tau^{\prime}\right)\right\rangle-N I_{N} \leqq 0,
$$

where $l=K N^{-1 / 2}$. If $\hat{F}_{L}^{(i)}$ satisfies that

$$
\left\{x \mid \hat{F}_{L}^{(i)}(x)>0\right\} \text { is compact, }
$$

then for $\beta>0$ and $J=1$ (3.32) holds with $V$ defined by

$$
V_{i}\left(F, K, L, \tau, \tau^{\prime}\right)=M^{-1} \sum_{\alpha=1}^{M}\left[\hat{F}_{L, \tau}^{(i)}\left(y_{i \alpha}\right)-\hat{F}^{(i)}\left(y_{i}\right) 1\left(\left|y_{i}\right|<\tau^{\prime}\right)\right] .
$$

Proof. Let us start with the proof of (3.32). As in Lemma 3.3, we can use the logarithmic Sobolev inequality to have

$$
\begin{aligned}
& \beta N^{1 / 2}\langle J, V\rangle-N I_{N} \\
& \quad \leqq \sup _{i} \sup _{\left|y_{t}\right|<\tau^{\prime}} N^{1 / 2} l^{-3}\left\{\log \int \exp \left[\beta l^{3} M^{-1} \sum_{q=1}^{M} \hat{F}_{L, \tau}^{(i)}\left(y_{i q}\right)\right] \Phi_{K, y} d x-\beta l^{3} \hat{F}^{(i)}\left(y_{i}\right)\right\} .
\end{aligned}
$$

Again as in Lemma 3.3, we shall bound (3.35) only for $i=0$ and omit all indices $i$.

Note that the integration in (3.35) can be thought of as integration over $y_{q}$ with $y_{q}$ identically distributed according to the density

$$
\exp \left[-(2 L+1) h_{L}\left(y_{q}\right)\right] \equiv \int \delta\left(\bar{x}-y_{q}\right) \Phi_{L}(d x) .
$$

By Brascamp-Lieb [1] theorem $h_{L}$ is strictly convex with $\gamma_{2}>h_{L}^{\prime \prime}>\gamma_{1}$. We claim that (3.35) can be bounded by (assume $\beta>0$ for simplicity of notation)

$$
\begin{aligned}
& \beta N^{1 / 2}\left\{\left\langle\hat{F}_{L, \tau}\right\rangle_{y}+M^{-1} G_{1}\left(\hat{F}_{L, \tau}, y\right)+\beta l^{3} G_{2}\left(\hat{F}_{L, \tau}, y\right) M^{-1}\right. \\
& \left.\quad-\hat{F}(y)+O\left[\left(1+\beta^{2} l^{6}\right) M^{-2} L^{-1 / 2}\right]\right\},
\end{aligned}
$$


where the expectation is defined by

$$
\langle f\rangle_{y}=M(\lambda)^{-1} \int f(z) \exp \left[(2 L+1)\left(\lambda z-h_{L}(z)\right)\right] d z .
$$

Here $M(\lambda)$ denotes the normalization constant and $\lambda$ is chosen such that $\langle z\rangle_{y}=y$. As in Corollary 3.2 we also have

$$
\begin{aligned}
M^{-1} \sum_{q=1}^{M} \int \hat{F}_{L, \tau}\left(y_{q}\right) \Phi_{K, y}(d x)= & \left\langle\hat{F}_{L, \tau}\right\rangle_{y}+M^{-1} G_{1}\left(\hat{F}_{L, \tau}, y\right) \\
& +O\left(M^{-2} G_{2}\left(\hat{F}_{L, \tau}, y\right)\right)+O\left(M^{-2} L^{-1 / 2}\right) .
\end{aligned}
$$

Note that $G_{1}$ and $G_{2}$ are defined in (3.7) with expectation defined in (3.38).

To prove (3.37), let us first make a few comments and normalization. First of all we shall prove (3.37) with $\hat{F}_{L, \tau}$ replaced by any bounded smooth function $U$. Recall that $y$ is the average of $y_{q}$. By translation we can assume that $y=0$. By changing $h_{L}\left(y_{q}\right)$ to $h_{L}\left(y_{q}\right)+\lambda y_{q}-\log M(\lambda)$, we can assume that $\left\langle y_{q}\right\rangle=0=y$. For simplicity, we shall assume that $\lambda=0$. Finally one can check that if (3.37) holds for some function $U$, it holds with $U$ replaced by $U+$ const. Hence we can assume the normalization condition that $U(0)=0$. Clearly $U$ satisfies the trivial bound (recall that $U$ is bounded)

$$
U(z) \leqq \text { const. }|z| \text {. }
$$

Let $w_{q}$ be the real variable defined by $w_{q}=L^{1 / 2} y_{q}$ and let $\xi(w)$ be defined by $\xi(w)=$ $L^{1 / 2} U\left(w L^{-1 / 2}\right)$. Clearly $\xi(w) \leqq$ const. $\left(1+w^{2}\right)$ and $w_{q}$ has the density $\exp \left[-\psi\left(w_{q}\right)\right]$ with const. $\gamma_{1}<\psi^{\prime \prime}(z)<$ const. $\gamma_{2}$ for all $z \in \mathbb{R}$. Hence the expectation in (3.35) becomes

$$
E\left[\exp \left[\beta l^{3} L^{-1 / 2} M^{-1} \sum_{\alpha=1}^{M} \xi\left(w_{q}\right)\right] \mid A v w_{q}=0\right] .
$$

We can thus apply Lemma 3.1. By changing the variable back to $y_{q}$ we conclude (3.37). Note that there is an extra factor $L^{-1 / 2}$ in the error term of (3.37) which comes from the $L^{-1 / 2}$ factor in the exponent of the above expectation.

To prove (3.37) for $\hat{F}_{L, \tau}$, we only have to check where we have used the assumption of smoothness of $U$. Clearly, we need it only to have that $U$ is bounded by const. $|z|$. For this purpose, the smoothness of $U$ in a neighborhood of $y$ suffices. By assumption $|y|<\tau^{\prime}<\tau$ and thus $\hat{F}_{L, \tau}$ is smooth in a neighborhood of $y$. Therefore the above argument works for $\hat{F}_{L, \tau}$ as well. We now continue our proof of (3.32).

The expectation in (3.38) can be computed asymptotically since $h_{L}$ is strictly convex and $L$ is large. Suppose that the unique minimum of $h_{L}(z)-\lambda z$ occurs at $z=z_{0}$. If $f$ is smooth in a neighborhood of $z_{0}$ and grows at most polynomially, we have

$$
\left|\langle f\rangle_{y}-\left[f\left(z_{0}\right)+\frac{1}{2} f^{\prime \prime}\left(z_{0}\right) h^{\prime \prime}\left(z_{0}\right)(2 L+1)^{-1}\right]\right| \leqq \text { const. } L^{-3 / 2} .
$$

Indeed, if $g$ is also of polynomial growth and $g$ equals $f$ in a neighborhood of $z_{0}$, then $\langle g\rangle_{y}$ and $\langle f\rangle_{y}$ is arbitrarily close in the sense that

$$
\left|\langle f\rangle_{y}-\langle g\rangle_{y}\right| \leqq c_{n} L^{-n}, \quad \forall n \in \mathbb{N} .
$$

By considering $f(z)=z$ we have that

$$
\left|y-z_{0}\right| \leqq \text { const. } L^{-3 / 2} \text {. }
$$

Since $|y|<\tau^{\prime}<\tau$, all the expectations in (3.37) and (3.39) involving $\hat{F}_{L, \tau}$ can be 
replaced by $\hat{F}_{L}(\cdot)$ with arbitrary small errors. But by definition

$$
M^{-1} \sum_{q=1}^{M} \int \hat{F}_{L}\left(y_{q}\right) \Phi_{K, y}(d x)=\hat{F}_{K}(y) .
$$

By Corollary 3.2, $\left|\hat{F}_{K}(y)-\hat{F}(y)\right| \leqq$ const. $K^{-1}$ and thus we can bound (3.37) by

$$
\text { const. }(\beta, l) N^{1 / 2}\left[M^{-2} L^{-1 / 2}+M^{-1}\left|G_{2}\left(\hat{F}_{L}, y\right)\right|\right]+\beta N^{1 / 2} K^{-1} \text {. }
$$

A simple computation shows that the zeroth and first order $\left(L^{-1}\right)$ terms vanish in $G_{2}$. Thus $\left|G_{2}\right| \leqq$ const. $L^{-3 / 2}$ and we can bound (3.43) by

$$
C(\beta, l)\left[N^{1 / 2} M^{-2} L^{-1 / 2}+N^{1 / 2} M^{-1} L^{-3 / 2}\right]+\beta N^{1 / 2} K^{-1} \leqq C(\beta, l) N^{-1 / 20}+l^{-1},
$$

where we have used the assumption that $L \sim N^{3 / 10}$. This concludes (3.32).

To prove (3.32) assuming (3.33) and (3.34) we only have to consider the case $|y| \geqq \tau^{\prime}$. Since $\tau^{\prime}$ is large, by assumption (3.33) we can find a bounded smooth function $W^{(i)}$ such that $\hat{F}_{L, \tau}^{(i)}(z) \leqq W^{(i)}(z), W^{(i)}(z)=\hat{F}_{L, \tau}(z)$ for $|z|<\tau^{\prime}-2$ and $W(z)=0$ for $|z| \geqq \tau^{\prime}-1$. Clearly we obtain an upper bound of $(3.35)$ by replacing $\hat{F}_{L, \tau}^{(i)}$ by $W^{(i)}$. We then proceed as in the previous proof. Note that we need the error term in (3.37) to be uniform with respect to $y$, i.e. it is bounded by const. $\left(1+\beta^{2} l^{6}\right) M^{-2} L^{-1 / 2}$ with the const. independent of $y$. This is not difficult to see because we can make a translation to have $y=0$ while maintaining the assumptions of Lemma 3.1: The assumption (3.3) is satisfied independent of translations of $y$ because $W_{\tau}$ is bounded; the convexity bound is also satisfied independent of translations since $h_{L}^{\prime \prime}(y)>\gamma^{\prime}$ for all $y$. Hence it remains to bound the first three terms in (3.37) (the term $\hat{F}$ vanishes in this case). As in the previous case, they can be approximated by using (3.40) (3.42). But since $|y| \geqq \tau^{\prime}$, we have, by (3.42) and our construction of $W$, that $W(z)=0$ in a neighborhood of $z_{0}$. This implies that all expectations involving $W$ are zero up to, say, $L^{-100}$. Therefore, we have the same bound as before and have proved (3.32) assuming (3.33) and (3.34).

Theorem 3.5. Suppose that $\phi$ satisfies the convexity bound (A1):

(i) If $F$ satisfies (3.3) then for all $\beta, J$ and $K=l N^{1 / 2}$ with $l$ a fixed constant,

$$
t^{-1} N^{-1} \log E_{\mathrm{eq}}^{N}\left[\exp \left\{\beta N^{3 / 2} \int_{0}^{t}\langle J, V(F, K, \tau)(s)\rangle d s\right\}\right] \leqq \text { const. }(l)\left[\beta^{2}\|J\|_{\infty}^{2}+1\right]
$$

where $V$ is defined in (3.15).

(ii) If the initial entropy satisfies the linear bound (A2) and $F^{(i)}$ satisfies (3.3) then

$$
\varlimsup_{N \rightarrow \infty} E_{\text {non }}^{N}\left[N^{1 / 2} \int_{0}^{t}\langle 1, V(F, K, \tau)(s)\rangle d s\right] \leqq 0,
$$

provided that $K$ satisfies $\lim _{N \rightarrow \infty} K N^{-1 / 2}=0$ and $\lim _{N \rightarrow \infty} N^{1 / 2} K^{-2}=0$. Furthermore, if $\hat{F}_{L}^{(i)}$ satisfies (3.33) then for $\tau^{\prime}<\tau$ large enough, $L \sim N^{3 / 10}$ and $V$ defined in (3.34)

$$
\varlimsup_{l \rightarrow \infty} \varlimsup_{N \rightarrow \infty} N^{1 / 2} E_{\text {non }}^{N}\left\{\int_{0}^{t}\left\langle 1, V\left(F, K, L, \tau, \tau^{\prime}\right)\right\rangle(s) d s\right\} \leqq 0 .
$$

Here F may depend explicitly on the time in both (3.46) and (3.47). 
(iii) If the initial entropy satisfies the linear bound (A2) and $F^{(i)}(x, s)=F(x)$ satisfies (3.3) then

$$
\varlimsup_{N \rightarrow \infty} \sup _{\|J\|_{\infty}<1} E_{\text {non }}^{N}\left\{N^{1 / 2}\left|\int_{0}^{t}\langle J, V(F, K, \tau)(s)\rangle d s\right|\right\}=0
$$

provided that $K$ satisfies $\lim _{N \rightarrow \infty} K N^{-1 / 2}=0$ and $\lim _{N \rightarrow \infty} N^{1 / 2} K^{-2}=0$. Also for $\tau^{\prime}<\tau$ large enough, $L \sim N^{3 / 10}$ and $V$ defined in (3.30)

$$
\varlimsup_{l \rightarrow \infty} \varlimsup_{N \rightarrow \infty} \sup _{\|J\|_{\infty}<1} E_{\text {non }}^{N}\left\{N^{1 / 2}\left|\int_{0}^{t}\left\langle J, V\left(F, K, L, \tau, \tau^{\prime}\right)(s) d s\right\rangle\right|\right\}=0 .
$$

Here $F$ is independent of time in either (3.48) or (3.49).

Proof. The bound (3.45) is an immediate corollary of (2.14) and Lemma 3.3. For (3.46) let us integrate (3.17) to have

$$
\begin{aligned}
& E_{\text {non }}^{N}\left\{N^{1 / 2} \int_{0}^{t}\langle 1, V(F, K, \tau)\rangle(s) d s\right\} \\
& \leqq \beta^{-1} \int_{0}^{t} N I_{N}\left(f(s)^{1 / 2}\right) d s+\text { const. }\left[\beta l^{2}+l^{-2} N^{-1 / 2}\right] t,
\end{aligned}
$$

where $f(s)$ is the density of the system at time $s$. But it is well known that the Dirichlet form of $f(s)$ satisfies the bound [6] (recall also the assumption (A2))

$$
N^{2} \int_{0}^{t} I_{N}\left(f(s)^{1 / 2}\right) d s \leqq H_{N}\left(f_{N}(0)\right) \leqq C N .
$$

Hence (3.46) follows by taking $\beta \rightarrow \infty$ after $N \rightarrow \infty$. (Note that $l=K N^{-1 / 2} \rightarrow 0$ by assumption). By a similar argument but using Lemma 3.4 one can prove (3.47).

Finally we have to prove (iii). By entropy inequality (2.12) we have for any $X$,

$$
E_{\text {non }}^{N}\left[\left|\int_{0}^{t} X\right|\right] \leqq \beta^{-1} N^{-1} \log E_{\mathrm{eq}}^{N}\left[\exp \left\{\beta N\left|\int_{0}^{t} X\right|\right\}\right]+H\left(f_{N}(0)\right) / \beta N
$$

Using the elementary inequality $e^{|a|} \leqq e^{a}+e^{-a}$ we only have to bound (3.51) with $\left|\int_{0}^{t} X\right|$ replaced by $\int_{0}^{t} X$ or $-\int_{0}^{t} X$. Hence it reduces to an eigenvalue problem again. We can now proceed as in the proof of (ii) and conclude (iii).

Lemma 3.6. Suppose the assumption (A1) and (A2) in Theorem 1 is satisfied. Then for any family of functions $\left\{F^{(i)}\right\}$ with $F^{(i)}$ satisfying (3.3) we have the following bound.

$$
E_{\text {non }}^{N}\left\{N^{-1} \sum_{i=1}^{N}\left|F^{(i)}\left(X_{i}(t)\right)\right|\right\} \leqq \text { const. }
$$

Here the constant depends on the constants in (3.3) and (A2) but is independent of $N$.

Proof. By entropy inequality (2.12) we only have to prove that $(\beta>0)$

$$
\log E_{\mathrm{eq}}^{N}\left\{\exp \left[\beta \sum_{i=1}^{N}\left|F^{(i)}\left(X_{i}(t)\right)\right|\right\} \leqq C(\beta) N\right.
$$


Since the equilibrium measure is product, one needs only to check the integrability of $\exp \left[\beta\left|F^{(i)}\left(X_{i}\right)\right|\right]$ with respect to $\exp (-\phi(x)) d x$. By (A1) and (3.3), this can be achieved if $\beta$ is chosen small enough.

\section{Proof of Theorem 2}

Our goal in this section is to prove Theorem 2. Indeed, we shall prove the following straightened version of Theorem 2 .

Lemma 4.1. Suppose that assumptions (A1), (A2) and (A3) are satisfied. As before, $L \sim N^{3 / 10}$ and $2 K+1=(2 L+1) M$. Let $Y_{\tau}$ and $U_{\tau}$ be defined by

$$
\begin{aligned}
& Y_{\tau, i}(s)=(2 L+1)^{-1} \sum_{|j-i| \leqq L} x_{j}^{2}(s) 1\left(\left|\bar{x}_{i, L}(s)\right| \geqq \tau\right) \\
& U_{\tau, i}(s)=\left(\bar{x}_{i, K}(s)-m_{i}(s)\right)^{2} 1\left(\left|\bar{x}_{i, K}(s)\right|<\tau\right) .
\end{aligned}
$$

Then for $\tau^{\prime}$ and $\tau$ large enough with $\tau^{\prime}<\tau$ we have

$$
\begin{aligned}
& E_{\text {non }}^{N}\left[(1 / 3) N^{-1 / 2}\left\|\tilde{\zeta}^{N}\right\|_{-1}^{2}(t)+\gamma_{1} N^{1 / 2} \int_{0}^{t}\left\langle 1, U_{\tau^{\prime}}+Y_{\tau}\right\rangle(s) d s\right] \\
& \leqq N^{-1 / 2} E_{\text {non }}^{N}\left[\left\|\tilde{\zeta}^{N}\right\|_{-1}^{2}(0)\right]+C_{N, K}, \\
& \varlimsup_{l \rightarrow \infty} \varlimsup_{N \rightarrow \infty} C_{N, K}=0, \quad l=N^{-1 / 2} K .
\end{aligned}
$$

Here $\gamma_{1}$ is defined in (A1) and $\tilde{\zeta}$ is defined in (A3) of Sect. 1.

Proof. By the SDE (1.3) we have (omitting all superscripts $N$ )

$$
d \tilde{\zeta}(t)=\frac{1}{2} N^{5 / 2} \Delta\left\{\phi^{\prime}\left(x_{i}(t)\right)-h^{\prime}\left(m_{i}(t)\right)\right\} d t+N^{3 / 2}\left\{d \beta_{i}(t)-d \beta_{i+1}(t)\right\} .
$$

A simple computation using Ito's formula then yields that

$$
\begin{aligned}
E_{\text {non }} & {\left[N^{-1 / 2}\|\tilde{\zeta}\|_{-1}^{2}(t)+2\langle\bar{x}-\bar{m}, \tilde{\zeta}\rangle_{-1}(t)\right] } \\
= & E_{\text {non }}\left[N^{-1 / 2}\|\tilde{\zeta}\|_{-1}^{2}(0)+2\langle\bar{x}-\bar{m}, \tilde{\zeta}\rangle_{-1}(0)\right] \\
& +E_{\text {non }}\left[\int_{0}^{t} d s N^{-1 / 2} \sum_{i=1}^{N}\left\{1-\left(\phi^{\prime}\left(x_{i}(s)\right)-h^{\prime}\left(m_{i}(s)\right)\right)\left(x_{i}(s)-m_{i}(s)\right)\right\}\right] .
\end{aligned}
$$

By assumption $E_{\text {non }}\left[(\bar{x}-\bar{m})^{2}\right]=o\left(N^{-1 / 2}\right)$ and thus

$$
\begin{aligned}
(1 / 2) N^{-1 / 2} E_{\text {non }}\left[\|\tilde{\zeta}\|_{-1}^{2}(s)\right]+o(1) & \leqq N^{-1 / 2} E_{\text {non }}\left[\|\tilde{\zeta}\|_{-1}^{2}(s)+2\langle\bar{x}-\bar{m}, \tilde{\zeta}\rangle_{-1}(s)\right] \\
& \leqq 3 / 2 N^{-1 / 2} E_{\text {son }}\left[\|\tilde{\zeta}\|_{-1}^{2}(s)\right]+o(1) .
\end{aligned}
$$

For each term in the summation of (4.5) we replace it by its local average, namely replace $\left(\phi^{\prime}\left(x_{i}\right)-h^{\prime}\left(m_{i}\right)\right)\left(x_{i}-m_{i}\right)$ by $(2 L+1)^{-1} \sum_{|j-i| \leqq L}\left(\phi^{\prime}\left(x_{j}\right)-h^{\prime}\left(m_{i}\right)\right)\left(x_{j}-m_{i}\right)$. This
produces an error term

$$
\begin{aligned}
& E_{\mathrm{non}}^{N}\left[\int _ { 0 } ^ { t } d t \left[N ^ { - 1 / 2 } \sum _ { i = 1 } ^ { N } ( 2 L + 1 ) ^ { - 1 } \sum _ { | j - i | \leqq L } \left\{\left(h^{\prime}\left(m_{j}(t)\right) m_{j}(t)-h^{\prime}\left(m_{i}(t)\right) m_{i}(t)\right)\right.\right.\right. \\
& \left.\left.\left.\quad+\left(h^{\prime}\left(m_{i}(t)\right)-h^{\prime}\left(m_{j}(t)\right)\right) x_{i}(t)+\left(m_{i}(t)-m_{j}(t)\right) \phi^{\prime}\left(x_{i}(t)\right)\right\}\right]\right] .
\end{aligned}
$$


Since $m$ is a "smooth" solution to (2.1), the coefficients of $x_{i}$ and $\phi^{\prime}\left(x_{i}\right)$ are $O\left(L^{2} N^{-2}\right)$. Similarly the first term of $(4.7)$ is also of $O\left(N^{-1 / 2}\right)$. Thus by Lemma 3.6 we can bound (4.7) by $C / N^{1 / 2}$.

Combining (4.5) with (4.7) we have

$$
\begin{aligned}
1 / 3 N^{-1 / 2} E_{\mathrm{non}}^{N}\left[\|\tilde{\zeta}\|_{-1}^{2}(t)\right] \leqq N^{-1 / 2} E_{\mathrm{non}}^{N}\left[\|\tilde{\zeta}\|_{-1}^{2}(0)\right] \\
+E_{\mathrm{non}}^{N}\left[\int_{0}^{t} d s\left\{N^{-1 / 2} \sum_{i=1}^{N}(2 L+1)^{-1} \sum_{|j-i| \leqq L} F^{(i)}\left(x_{j}(s), s\right)\right\}\right]+o(1)
\end{aligned}
$$

where $F^{(i)}(x, s)$ is defined by

$$
F^{(i)}(x, s)=1-\left(\phi^{\prime}(x)-h^{\prime}\left(m_{i}(s)\right)\left(x-m_{i}(s)\right)\right) .
$$
Decompose $(2 L+1)^{-1} \sum_{|j-i| \leqq L} F^{(i)}\left(x_{j}, s\right)$ as $W_{i}^{(1)}+W_{i}^{(2)}+W_{i}^{(3)}$, where $W_{i}^{(\sigma)}$ is
defined by

$$
\begin{aligned}
& W_{i}^{(1)}=(2 L+1)^{-1} \sum_{|j-i| \leqq L}\left[F^{(i)}\left(x_{j}, s\right)-\hat{F}_{L}^{(i)}\left(\bar{x}_{i, L}, s\right)\right] 1\left(\left|\bar{x}_{i, L}\right|<\tau\right), \\
& W_{i}^{(2)}=(2 L+1)^{-1} \sum_{|j-i| \leqq L} F^{(i)}\left(x_{j}, s\right) 1\left(\left|\bar{x}_{i, L}\right| \geqq \tau\right), \\
& W_{i}^{(3)}=\hat{F}_{L}^{(i)}\left(\bar{x}_{i, L}, s\right) 1\left(\left|\bar{x}_{i, L}\right|<\tau\right) .
\end{aligned}
$$

By (3.46) we can bound $W^{(1)}$ by

$$
\varlimsup_{N \rightarrow \infty} N^{1 / 2} E_{\text {non }}^{N}\left[\int_{0}^{t}\left\langle 1, W^{(1)}\right\rangle(s) d s\right] \leqq 0 .
$$

Also, by convexity of $\phi$ we have for $\tau$ sufficiently large

$$
W_{i}^{(2)}(s)<-\frac{\gamma_{1}}{2} Y_{\tau, i}(s)
$$

Therefore,

$$
\begin{gathered}
1 / 3 N^{-1 / 2} E_{\text {non }}\left[\|\tilde{\zeta}\|_{-1}^{2}(t)\right] \leqq N^{-1 / 2} E_{\text {non }}^{N}\left[\|\tilde{\zeta}\|_{-1}^{2}(0)\right]+o(1)-\left(\gamma_{1} / 2\right) N^{1 / 2} \\
\cdot E_{\text {non }}^{N}\left[\int_{0}^{t}\left\langle 1, Y_{\tau}\right\rangle(s) d s\right]+E_{\text {non }}^{N}\left[\int_{0}^{t} N^{-1 / 2} \sum_{i=1}^{N} W_{i}^{(3)}\left(\bar{x}_{i, L}(s)\right) d s\right] .
\end{gathered}
$$

Decompose the last term as

$$
\begin{aligned}
N^{-1 / 2} \sum_{i=1}^{N} W_{i}^{(3)}\left(\bar{x}_{i, L}(s)\right)= & N^{1 / 2}\left\langle 1, V\left(F, K, L, \tau, \tau^{\prime}\right)\right\rangle \\
& +N^{-1 / 2} \sum_{i=1}^{N} W_{i}^{(4)}+N^{-1 / 2} \sum_{i=1}^{N} \hat{F}^{(i)}\left(\left|\bar{x}_{i, K}\right|<\tau^{\prime}\right),
\end{aligned}
$$

where $V$ is defined in (3.34) and $W^{(4)}$ is defined by (with $y_{i \alpha}$ defined in (3.31))

$$
W_{i}^{(4)}=M^{-1} \sum_{\alpha=1}^{M}\left[\hat{F}_{L}^{(i)}\left(y_{i \alpha}\right) 1\left(\left|y_{i \alpha}\right|<\tau\right)-\hat{F}_{L}^{(i)}\left(\bar{x}_{i, L}\right) 1\left(\left|\bar{x}_{i L}\right|<\tau\right)\right] .
$$


As in (4.7) one can check that $W^{(4)}$ is negligibly small in the sense that

$$
\varlimsup_{l \rightarrow \infty} \varlimsup_{N \rightarrow \infty} N^{1 / 2} E_{\text {non }}^{N} \int_{0}^{t}\left|\left\langle 1, W^{(4)}(s)\right\rangle\right| d s=0 .
$$

The expectation of the first term on the right side of (4.13) is also negligibly small by (3.47). Finally the last term can be bounded by using the fact that

$$
\begin{aligned}
\hat{F}^{(i)}(y) & =M(\lambda)^{-1} \int F^{(i)}(y) e^{\lambda x-\phi(x)} d x \\
& =-\left(y-m_{i}\right)\left(h^{\prime}(y)-h^{\prime}(m)\right) \leqq-\gamma_{1}(y-m)^{2},
\end{aligned}
$$

where we have used the convexity of $h$. Combining $(4.8) \sim(4.15)$, we have proved Lemma 4.1.

Proof of Theorem 2. By Lemma 4.1 we have for all $\tau$ large enough,

$$
\begin{aligned}
& \varlimsup_{l \rightarrow \infty} \varlimsup_{N \rightarrow \infty} N^{1 / 2} E_{\text {non }}^{N}\left[\int_{0}^{t}\left\langle 1, U_{\tau}\right\rangle(s) d s\right]=0, \\
& \varlimsup_{l \rightarrow \infty} \varlimsup_{N \rightarrow \infty} N^{1 / 2} E_{\text {non }}^{N}\left[\int_{0}^{t}\left\langle 1, Y_{\tau}\right\rangle(s) d s\right]=0 .
\end{aligned}
$$

We claim that for $\tau$ large enough

$$
3\left\langle 1, Y_{\tau / 3}\right\rangle \geqq\left\langle 1, U-U_{\tau}\right\rangle, \quad U=U_{\tau=\infty} .
$$

This follows from the inequalities (with $\tau \geqq m_{i}$ and $y_{i \alpha}$ defined in (3.31))

$$
\begin{aligned}
& \left(\bar{x}_{i, K}-m_{i}\right)^{2} 1\left(\left|\bar{x}_{i, K}\right| \geqq \tau\right)<\left[3 \bar{x}_{i, K}^{2}-\tau^{2}\right] 1\left(\left|\bar{x}_{i, K}\right| \geqq \tau\right) \\
& \leqq M^{-1} \sum_{\alpha=1}^{M}\left[3 y_{i \alpha}^{2}-\tau^{2}\right] 1\left(\left|\bar{x}_{i, K}\right| \geqq \tau\right) \\
& \leqq 3 M^{-1} \sum_{\alpha=1}^{M}\left[y_{i \alpha}^{2} 1\left(\left|y_{i \alpha}\right| \geqq \tau / 3\right)\right] 1\left(\left|\bar{x}_{t, K}\right| \geqq \tau\right)
\end{aligned}
$$

and that

$$
(2 L+1)^{-1} \sum_{|j-i| \leqq L} x_{j}^{2} \geqq \bar{x}_{i, L}^{2}
$$

Theorem 2 then follows from (4.16)-(4.20) and Lemma 4.1.

\section{Proof of Theorem 1}

Recall that $\zeta$ satisfies $\operatorname{SDE}$ (2.2). Let $\Omega$ and $Z^{N}$ be defined by

$$
\begin{gathered}
\Omega_{i}(s)=N^{1 / 2}\left[\phi^{\prime}\left(x_{i}(s)\right)-h^{\prime}\left(m_{i}(s)\right)-h^{\prime \prime}\left(m_{i}(s)\right)\left(x_{i}(s)-m_{i}(s)\right)\right], \\
Z^{N}(t)=\frac{1}{2} \int_{0}^{t} U^{N}(t, s) N^{2} \Delta \Omega(s) d s,
\end{gathered}
$$


where $U^{N}$ is the semigroup of the equation

$$
\frac{\partial f}{\partial t}=\frac{1}{2} N^{2} \Delta\left[h^{\prime \prime}\left(m_{i}(t)\right) f\right] .
$$

Then we can integrate $(2.2)$ to have

$$
\zeta^{N}(t)=U^{N}(t, 0) \zeta^{N}(0)+\int_{0}^{t} U^{N}(t, s) N^{3 / 2} \nabla d \beta(s)+Z^{N}(t) .
$$

Let $|J|_{\alpha}$ be defined by

$$
|J|_{\alpha}=\sum_{i=0}^{\alpha}\left\|J^{(i)}\right\|_{\infty}
$$

where $J^{(i)}$ is the (scaled) lattice difference operator of order $i$, e.g. $J^{(1)}(i / N)=$ $N[J((i+1) / N)-J(i / N)]$. We have the following lemma.

Lemma 5.1. Suppose that assumptions (A1)-(A3) are satisfied. Then

$$
\lim _{l \rightarrow \infty} \lim _{N \rightarrow \infty} \sup _{|J|_{4}<1} E_{\text {non }}^{N}\left[\left|\left\langle J, Z^{N}(t)\right\rangle\right|\right]=0 .
$$

Proof. We first claim that it suffices to show that

$$
\lim _{l \rightarrow \infty} \lim _{N \rightarrow \infty} \sup _{|J|_{2} \leqq 1} E_{\text {non }}^{N}\left[\left|\int_{0}^{t}\langle J, \Omega(s)\rangle d s\right|\right]=0
$$

uniformly on compact $t$-intervals. To see this we integrate the $t$-integration by parts,

$$
\int_{0}^{t}\left\langle J, U(t, s) N^{2} \Delta \Omega(s)\right\rangle d s=\int_{0}^{t}\left\langle N^{2} \Delta J, \Omega(s)\right\rangle d s-\int_{0}^{t} \int_{0}^{s}\langle\tilde{J}, \Omega(\tau)\rangle d \tau d s,
$$

where $\tilde{J}$ is given by

$$
\tilde{J}(t, s)=\frac{d}{d s}\left(N^{2} \Delta U^{*}(t, s) J\right)
$$

with $U^{*}$ denoting the adjoint of $U$. By parabolic regularity, $|\widetilde{J}|_{2} \leqq C|J|_{4}$. Thus by (5.7) each term on the right side vanishes after taking expectation. We now prove (5.7).

Let us decompose $\Omega$ as

$$
\Omega=\Omega^{(1)}+\Omega^{(2)}+\Omega^{(3)}+\Omega^{(4)},
$$

where $\Omega^{(\sigma)}$ is defined by (with $\tau^{\prime}$ denoting some large constant)

$$
\begin{aligned}
& \Omega_{i}^{(1)}=N^{1 / 2}\left[(2 K+1)^{-1} \sum_{|j-i| \leqq K} \phi^{\prime}\left(x_{j}\right)-h^{\prime}\left(y_{i}\right) 1\left(\left|y_{i}\right|<\tau^{\prime}\right)\right], \quad y_{i} \equiv \bar{x}_{i, k}, \\
& \Omega_{i}^{(2)}=N^{1 / 2}\left[h^{\prime}\left(y_{i}\right)-h^{\prime}\left(m_{i}\right)-h^{\prime \prime}\left(m_{i}\right)\left(y_{i}-m_{i}\right)\right] 1\left(\left|y_{i}\right|<\tau^{\prime}\right), \\
& \Omega_{i}^{(3)}=-N^{1 / 2}\left[h^{\prime}\left(m_{i}\right)+h^{\prime \prime}\left(m_{i}\right)\left(y_{i}-m_{i}\right)\right] 1\left(\left|y_{i}\right| \geqq \tau^{\prime}\right), \\
& \Omega_{i}^{(4)}=N^{1 / 2}\left[(2 K+1)^{-1} \sum_{|j-i| \leqq K}\left(\phi^{\prime}\left(x_{i}\right)-\phi^{\prime}\left(x_{j}\right)\right)+h^{\prime \prime}\left(m_{i}\right)\left(y_{i}-x_{i}\right)\right] .
\end{aligned}
$$


By summation by parts, regularity of solutions to (2.1) and Lemma 3.6 we can bound $\Omega^{(4)}$ by

$$
\varlimsup_{l \rightarrow \infty} \varlimsup_{N \rightarrow \infty} E_{\text {non }}^{N}\left[\sup _{|J|_{2}<1} \int_{0}^{t}\left|\left\langle J, \Omega^{(4)}\right\rangle\right|(s) d s\right]=0 .
$$

By the Taylor theorem,

$$
\left|\Omega_{i}^{(2)}\right| \leqq \text { const. } N^{1 / 2}\left(y_{i}-m_{i}\right)^{2} 1\left(\left|y_{i}\right|<\tau^{\prime}\right) .
$$

Hence by Theorem 2,

$$
\varlimsup_{l \rightarrow \infty} \varlimsup_{N \rightarrow \infty} E_{\text {non }}^{N}\left[\sup _{|J|_{2}<1} \int_{0}^{t}\left|\left\langle J, \Omega^{(2)}\right\rangle(s)\right| d s\right]=0 .
$$

To bound $\Omega^{(3)}$, note that by a similar argument given in (4.19)

$$
\begin{aligned}
\left|\Omega_{i}^{(3)}\right| & \leqq \text { const. } N^{1 / 2}\left[1+\left|y_{i}\right|\right] 1\left(\left|y_{i}\right|>\tau^{\prime}\right) \\
& \leqq \text { const. } N^{1 / 2} M^{-1} \sum_{\alpha=1}^{M} y_{i \alpha}^{2} 1\left(\left|y_{i \alpha}\right|>\tau^{\prime} / 3\right) 1\left(\left|y_{i}\right|>\tau^{\prime}\right) .
\end{aligned}
$$

Hence by Lemma 4.1

$$
\varlimsup_{l \rightarrow \infty} \varlimsup_{N \rightarrow \alpha} E_{\text {non }}^{N}\left[\sup _{|J|_{2}<1} \int_{0}^{t}\left|\left\langle J, \Omega^{(3)}\right\rangle(s)\right| d s\right]=0 .
$$

We now decompose $\Omega^{(1)}$ as $\Omega^{(6)}+\Omega^{(7)}+\Omega^{(8)}$ with $\Omega^{(\sigma)}$ defined by $\left(\tau^{\prime}<\tau\right.$ as in Theorem 3.5)

$$
\begin{aligned}
& \Omega_{i}^{(6)}=N^{1 / 2} M^{-1} \sum_{\alpha=1}^{M}(2 L+1)^{-1} \sum_{j \in \alpha(i)}\left[\phi^{\prime}\left(x_{j}\right)-h_{L}^{\prime}\left(y_{i \alpha}\right)\right] 1\left(\left|y_{i \alpha}\right|<\tau\right), \\
& \Omega_{i}^{(7)}=N^{1 / 2} M^{-1} \sum_{\alpha=1}^{M}(2 L+1)^{-1} \sum_{j \in \alpha(i)} \phi^{\prime}\left(x_{j}\right) 1\left(\left|y_{i \alpha}\right| \geqq \tau\right), \\
& \Omega_{i}^{(8)}=N^{1 / 2} M^{-1} \sum_{\alpha=1}^{M} h_{L}^{\prime}\left(y_{i \alpha}\right) 1\left(\left|y_{i \alpha}\right|<\tau\right)-h^{\prime}\left(y_{i}\right) 1\left(\left|y_{i}\right|<\tau^{\prime}\right),
\end{aligned}
$$

where we have followed the conventions of notations in (3.31). By (3.48),

$$
\varlimsup_{l \rightarrow \infty} \varlimsup_{N \rightarrow \infty} \sup _{|J|_{2}<1} E_{\text {non }}^{N}\left[\left|\int_{0}^{t}\left\langle J, \Omega^{(6)}\right\rangle(s) d s\right|\right]=0 .
$$

By assumption (A1), we can bound $\left\langle J, \Omega^{(7)}\right\rangle$ by const. $N^{1 / 2}\left\langle 1, Y_{\tau}\right\rangle$ if $\tau$ is sufficiently large. Thus

$$
\varlimsup_{l \rightarrow \infty} \varlimsup_{N \rightarrow \infty} E_{\text {non }}^{N}\left[\sup _{|J|_{2}<1} \int_{0}^{t}\left|\left\langle J, \Omega^{(7)}\right\rangle(s) d s\right|\right]=0 .
$$

To bound $\Omega^{(8)}$ we decompose it further into $\Omega^{(9)}+\Omega^{(10)}$ with

$$
\begin{aligned}
\Omega_{i}^{(9)} & =N^{1 / 2} M^{-1} \sum_{\alpha=1}^{M}\left[h_{L}^{\prime}\left(y_{i \alpha}\right) 1\left(\left|y_{i \alpha}\right|<\tau\right)-h^{\prime}\left(y_{i}\right)\right] 1\left(\left|y_{i}\right|<\tau^{\prime}\right), \\
\Omega_{i}^{(10)} & =N^{1 / 2} M^{-1} \sum_{\alpha=1}^{M} h_{L}^{\prime}\left(y_{i \alpha}\right) 1\left(\left|y_{i \alpha}\right|<\tau\right) 1\left(\left|y_{i}\right| \geqq \tau^{\prime}\right) .
\end{aligned}
$$


By (3.49),

$$
\varlimsup_{l \rightarrow \infty} \varlimsup_{N \rightarrow \infty} \sup _{|J|_{2}<1} E_{\text {non }}^{N}\left[\left|\int_{0}^{t}\left\langle J, \Omega^{(9)}\right\rangle(s) d s\right|\right]=0 .
$$

Hence it remains to bound $\Omega^{(10)}$. Since $\left\|h_{L}^{\prime}(\cdot) 1(|\cdot|<\tau)\right\|_{\infty}$ is bounded,

$$
\left|\Omega_{i}^{(10)}\right| \leqq \text { const. } N^{1 / 2}\left(y_{i}-m_{i}\right)^{2} .
$$

It follows from (1.17) that

$$
\varlimsup_{l \rightarrow \infty} \varlimsup_{N \rightarrow \infty} E_{\text {non }}^{N}\left[\sup _{|J|_{2}<1} \int_{0}^{t}\left|\left\langle J, \Omega^{(10)}\right\rangle(s)\right| d s\right]=0 .
$$

Combining (5.8)-(5.13), we have proved Lemma 5.1.

Lemma 5.2. Assume the assumptions (A1)-(A4). Let $Q^{N}$ be the distribution of the process $Z^{N}$. Then for $\alpha>9 / 2 Q^{N}$ converge weakly to the delta function concentrated at 0 in $C\left([0,1], H_{-\alpha}\right)$.

Proof. We only have to prove the tightness of the family $\left\{Q^{N}\right\}$ since by Lemma 5.1 the only possible limit point of $\left\{Q_{N}\right\}$ is the delta function concentrated at 0 .

Recall that the standard criterion for tightness is to prove that for all $\varepsilon>0$,

$$
\begin{gathered}
\lim _{\delta \rightarrow 0} \limsup _{N \rightarrow \infty} Q^{N}\left[\sup _{\substack{0<s<t \leqq 1 \\
|t-s|<\delta}}\left\|Z^{N}(s)-Z^{N}(t)\right\|_{-\alpha}>\varepsilon\right]=0, \\
\lim _{M \rightarrow \infty} \limsup _{N \rightarrow \infty} Q^{N}\left[\sup _{0<t \leqq 1}\left\|Z^{N}(t)\right\|_{-\alpha}>M\right]=0 .
\end{gathered}
$$

Since the proofs of (5.14) and (5.15) are similar, we shall only prove (5.14).

We first replace $\|X\|_{-\alpha}$ by $\sup _{\|J\|_{\alpha} \leqq 1}\langle J, x\rangle$. By definition we can write

$$
\left\langle J, Z^{N}(t)\right\rangle=\frac{N^{2}}{2} \int_{0}^{t}\left\langle\Delta U^{N}(t, \sigma)^{*} J, \Omega(\sigma)\right\rangle d \sigma .
$$

Integrate the right side of (5.16) by parts,

$$
\left\langle J, Z^{N}(t)\right\rangle=\int_{0}^{t}\left\langle J_{1}, \Omega(\tau)\right\rangle d \tau-\int_{0}^{t} d \tau\left\langle J_{2}(t, \tau), \int_{0}^{\tau} \Omega(\sigma) d \sigma\right\rangle,
$$

where $J_{1}$ and $J_{2}$ are given by

$$
J_{1}=\frac{1}{2} N^{2} \Delta J, \quad J_{2}(t, \tau)=\frac{1}{2} N^{2} \Delta \frac{d}{d \tau} U^{N}(t, \tau)^{*} J .
$$

Subtract $\left\langle J, Z^{N}(s)\right\rangle$ from (5.17),

$$
\begin{aligned}
\left\langle J, Z^{N}(t)-Z^{N}(s)\right\rangle= & \int_{s}^{t}\left\langle J_{1}, \Omega(\tau)\right\rangle d \tau-\int_{s}^{t} d \tau\left\langle J_{2}(t, \tau), \int_{0}^{\tau} \Omega(\sigma) d \sigma\right\rangle \\
& -\int_{0}^{s} d \tau\left\langle J_{2}(t, \tau)-J_{2}(s, \tau), \int_{0}^{\tau} \Omega(\sigma) d \sigma\right\rangle .
\end{aligned}
$$


From standard results of PDE we have

$$
\left\|J_{i}\right\|_{\alpha-2}<C\|J\|_{\alpha}, \quad i=1,2,
$$

and for all $\delta_{1}>0$ there is a $\delta$ such that

$$
\sup _{|s-t|<\delta} \sup _{\tau}\left\|J_{3}(s, t, \tau)\right\|_{\alpha-2}<\delta_{1}
$$

where $J_{3}(s, t, \tau)=J_{2}(t, \tau)-J_{2}(s, \tau)$. Thus the probability in (5.14) can be bounded by the sum of the following three terms:

$$
\begin{aligned}
& E_{\text {non }}^{N}\left\{\sup _{s, t} \sup _{\left\|J_{1}\right\|_{\alpha-2}<C}\left|\left\langle J_{1}, \int_{s}^{t} \Omega d \sigma\right\rangle\right|>\frac{\varepsilon}{3}\right\}, \\
& E_{\text {non }}^{N}\left\{\sup _{0 \leqq \tau \leqq 1} \sup _{\left\|J_{2}\right\|_{x-2}<C} \delta \cdot\left|\left\langle J_{2}, \int_{0}^{\tau} \Omega d \sigma\right\rangle\right|>\frac{\varepsilon}{3}\right\}, \\
& E_{\text {non }}^{N}\left\{\sup _{0 \leqq \tau} \sup _{\left\|J_{3}\right\|_{x-2}<\delta_{1}}\left|\left\langle J_{3}, \int_{0}^{\tau} \Omega d \sigma\right\rangle\right|>\frac{\varepsilon}{3}\right\} .
\end{aligned}
$$

Here the $\sup _{s, t}$ always means that $|s-t|<\delta$ and $0 \leqq s<t \leqq 1$. We shall prove that each of the above three terms tends to zero as $\delta \rightarrow 0$.

First we note that the event (5.24) is similar to the event in (5.23) by normalizing $J_{3}$, i.e. $J_{3} \rightarrow \delta_{1} J_{3}$. Hence we only have to prove that (5.22) and (5.23) tend to zero. But the events of (5.22) and (5.23) are in fact related by the inequality

$$
\sup _{0 \leqq \tau \leqq 1}\left|\left\langle J_{2}, \int_{0}^{\tau} \Omega d \sigma\right\rangle\right| \leqq \frac{1}{\delta} \sup _{s, t}\left|\left\langle J_{2}, \int_{\mathrm{s}}^{t} \Omega d \sigma\right\rangle\right| \text {. }
$$

Thus we only have to prove that (5.22) tends to zero as $\delta \rightarrow 0$. Since the range of $J_{1}$ is symmetric under the transformation $J_{1} \rightarrow-J_{1}$, we can take out the absolute value outside the integration of $\Omega$. We shall also drop the subscript of $J_{1}$ from now on.

Recall (5.8) that $\Omega$ can be decomposed as $\Omega=\sum_{\sigma=1}^{4} \Omega^{(\sigma)}$ with $\Omega^{(\sigma)}$ satisfies

$$
\varlimsup_{l \rightarrow \infty} \varlimsup_{N \rightarrow \infty} E_{\text {non }}^{N}\left[\sup _{|J|_{2}<1} \int_{0}^{t}\left|\left\langle J, \Omega^{(\sigma)}\right\rangle(s)\right| d s\right]=0, \quad \sigma=2,3,4 .
$$

By Sobolev's lemma, $|J|_{2} \leqq$ const. $\|J\|_{\alpha}$ if $\alpha>5 / 2$. Hence by choosing $l$ large enough, we can neglect $\Omega^{(\sigma)}, \sigma=2,3,4$ in bounding (5.22). Now we decompose $\Omega^{(1)}$ as $B+W$ with

$$
\begin{aligned}
& B_{i}=N^{1 / 2}(2 K+1)^{-1} \sum_{|j-i| \leqq K} \phi^{\prime}\left(x_{j}\right) 1\left(\left|y_{i}\right| \geqq \tau^{\prime}\right), \\
& W_{i}=N^{1 / 2}(2 K+1)^{-1} \sum_{|j-i| \leqq K}\left(\phi^{\prime}\left(x_{j}\right)-h^{\prime}\left(y_{i}\right)\right) 1\left(\left|y_{i}\right|<\tau^{\prime}\right) .
\end{aligned}
$$

As in the proof of Lemma $5.1,|\langle J, B\rangle|$ can be bounded by $N^{1 / 2}\left\langle 1, Y_{\tau^{\prime} / 2}\right\rangle$ and thus it satisfies $(5.25)$ with $\Omega^{(\sigma)}$ replaced by $B$. Hence we only have to prove that

$$
\lim _{\delta \rightarrow 0} \varlimsup_{N \rightarrow \infty} E_{\text {non }}^{N}\left\{\sup _{s, t} \sup _{\|J\|_{x-2}<C}\left\langle J, \int_{s}^{t} W(\sigma) d \sigma\right\rangle>\varepsilon / 5\right\}=0,
$$


where the integer $K$ is chosen to be $l \sqrt{N}$ with $l$ large enough such that the expectation in (5.25) is smaller than $\varepsilon / 100$.

$$
\begin{gathered}
\text { Replace } \sup _{\|J\|_{x-2}<C}\left\langle\int_{s}^{t} W, J\right\rangle \text { by } C\left\|\int_{s}^{t} W\right\|_{2-\alpha} \text {. Let } A \text { denote the event } \\
A=\sup _{s, t}\left\|\int_{s}^{t} W\right\|_{2-\alpha}>\frac{\varepsilon}{5} .
\end{gathered}
$$

By the standard entropy inequality [6]

$$
Q^{N}(A) \leqq\left[\log 2+H_{N}\right]\left[\log \left(1+P_{\mathrm{eq}}^{N}(A)^{-1}\right)\right]^{-1} .
$$

By assumption (A2) $H_{N} \leqq$ const. $N$. Hence it suffices to prove the superexponential bound of the event $A$ in the equilibrium, namely

$$
P_{\mathrm{eq}}^{N}(A) \leqq \exp [-N C(\delta)], \quad \lim _{\delta \rightarrow 0} C(\delta)=\infty .
$$

Since there is a sup appeared in the definition of $A$, we need the following lemma of Garsia [12]: For any continuous function $g$ we have

$$
\begin{aligned}
|g(t)-g(s)| & \leqq 8 \int_{0}^{t-s} \psi^{-1}\left(\frac{4 B}{u^{2}}\right) p(d u), \\
B & =\int_{0}^{T} \int_{0}^{T} \psi\left(\frac{|g(\sigma)-g(\tau)|}{p(|\sigma-\tau|)}\right) d \sigma d \tau,
\end{aligned}
$$

where $p$ and $\psi$ are strictly increasing continuous functions satisfying some technical conditions. The Garsia lemma also holds if $g$ is a continuous function with value in a Banach space. Let us choose

$$
\psi(x)=e^{N|x|}-1, \quad p(t)=\sqrt{t}, \quad g(t)=\int_{0}^{t} W(\sigma) d \sigma .
$$

Then

$$
\begin{gathered}
\sup _{\substack{0 \leqq s \leqq t \leqq 1 \\
|s-t|<\delta}}\left\|\int_{s}^{t} W(\sigma) d \sigma\right\|_{2-\alpha} \leqq 8 \int_{0}^{\delta} \psi^{-1}\left(\frac{4 B}{u^{2}}\right) p(d u), \\
B=-1+\int_{0}^{1} d s \int_{0}^{1} d t \exp \left\{N\left\|\int_{s}^{t} W(\sigma) d \sigma\right\|_{2-\alpha}|t-s|^{-1 / 2}\right\} .
\end{gathered}
$$

Since $\psi^{-1}(y)=N^{-1} \log (1+y)$, we can integrate the right side of (5.29) by parts

$$
\begin{aligned}
& \sup _{s, t}\left\|\int_{s}^{t} W(\sigma) d \sigma\right\|_{2-\alpha} \\
& \leqq 8 N^{-1}\left\{\log \left(1+4 B \delta^{-2}\right) \delta^{1 / 2}+8 \int_{0}^{\delta}\left[\left(B u^{-2}\right)\left(1+4 B u^{-2}\right)^{-1}\right] u^{-1 / 2} d u\right\} \\
& \leqq 8 N^{-1} \delta^{1 / 2}\left\{\log \left(1+4 B \delta^{-2}\right)+4\right\} .
\end{aligned}
$$

Hence

$$
P_{\mathrm{eq}}^{N}[A] \leqq P_{\mathrm{eq}}^{N}\left[B>C \delta^{2} \exp \left(N \varepsilon \delta^{-1 / 2}\right)\right] \leqq C E_{\mathrm{eq}}^{N}[B] \exp \left(-N \varepsilon \delta^{-1 / 2}\right) \delta^{-2} .
$$


If we can show that for each $l$ fixed

$$
E_{\mathrm{eq}}^{N}[B]<e^{C_{l} N},
$$

then (5.32) and (5.33) prove (5.27). So our final task is to prove (5.33). Since $l$ is fixed, we shall omit all $l$ indices.

First we use Schwartz inequality to bound the exponent in $B$, i.e.

$$
N\left\|\int_{s}^{t} W\right\|_{2-\alpha}(t-s)^{-1 / 2}<a^{2} N\left\|\int_{s}^{t} W\right\|_{2-\alpha}^{2}(t-s)^{-1}+N / a^{2} .
$$

Hence it suffices to prove that

$$
E_{\mathrm{eq}}^{N}\left[\exp \left\{\frac{a^{2}}{2} N(t-s)^{-1}\left\|\int_{s}^{t} W\right\|_{2-\alpha}^{2}\right\}\right]<e^{C N} .
$$

Let $d \mu_{\beta, \gamma}$ be the Gaussing measure on $H_{\beta}$ with covariance $S=\left(1-N^{2} \Delta\right)^{-\gamma}$. (Note that $N^{2} \Delta \sim \partial_{\theta}^{2}$.) The existence of such a measure with $\gamma>\frac{1}{2}$ is guaranteed by Prohorov Theorem [13]. Let \langle\rangle$_{\beta}$ denote the inner produce in $H_{\beta}$, i.e. $\langle f, g\rangle_{\beta}=\left\langle f,\left(1-N^{2} \Delta\right)^{\beta} g\right\rangle$. Then we have the identity [13]

$$
\begin{aligned}
\int d \mu_{\beta, \gamma}(J) \exp [\langle J, X\rangle] & =\int d \mu_{\beta, \gamma}(J) \exp \left[\left\langle J,\left(1-N^{2} \Delta\right)^{-\beta} X\right\rangle_{\beta}\right] \\
& =\exp \left[\frac{1}{2}\left\langle X, S\left(1-N^{2} \Delta\right)^{-2 \beta} X\right\rangle_{\beta}\right]=\exp \left[\frac{1}{2}\|X\|_{-\beta-\gamma}^{2}\right] .
\end{aligned}
$$

Since we are interested in bounding (5.34), we shall choose

$$
-\beta-\gamma=2-\alpha
$$

Thus (5.34) is equivalent to

$$
\int d \mu_{\beta, \gamma}(J) E_{\mathrm{eq}}^{N}\left[\exp \left\{a N^{1 / 2}(t-s)^{-1 / 2}\left\langle J, \int_{s}^{t} W\right\rangle\right\}\right]<e^{C N} .
$$

From (3.45)

$$
E_{\mathrm{eq}}^{N}\left[\exp \left(\left\{a N^{1 / 2}(t-s)^{-1 / 2}\left\langle J, \int_{s}^{t} W\right\rangle\right\}\right]<\exp \left\{C a^{2}\left(|J|_{1}^{2}+1\right)\right\} .\right.
$$

By Sobolev inequality, $|J|_{1}<C\|J\|_{2}$. Thus the left side of (5.37) is less than

$$
\int d \mu_{\beta, \gamma}(J) \exp \left\{C a^{2}\|J\|_{2}^{2}\right\}=\operatorname{det}\left(1-C a^{2}\left(1-N^{2} \Delta\right)^{-\beta+2-\gamma}\right)^{-1 / 2} .
$$

The last identity is a simple property of Gaussian measure. It holds with the determinant finite if $a$ is small enough and if $\beta \geqq 2$ (recall $\gamma>1 / 2$ ). Putting all this together we find that (5.37) holds if (recall (5.36))

$$
-\alpha+\beta+\gamma=-2, \quad \beta \geqq 2, \quad \gamma>\frac{1}{2} .
$$

Clearly, there exists at least one pair of $(\beta, \gamma)$ satisfying (5.40) if $\alpha>\frac{9}{2}$. This concludes Lemma 5.2.

Proof of Theorem 1. By Lemma 5.2 it remains to show the convergence of the first two terms on the right side of (5.4). We began by proving that

$$
\int_{0}^{t} U^{N}(t, s) N^{3 / 2} \nabla d \beta(s) \Rightarrow \int_{0}^{t} U^{\infty}(t, s) \partial_{\theta} d B(s) .
$$


First, it is easy to prove the tightness of the family of the left side of (5.41). Then since everything involved is Gaussian, we only have to check the convergence of the covariances. But this is straightforward and we omit the details. So our final task is to show that

$$
U^{N}(t, 0) \zeta^{N}(0) \Rightarrow U^{\infty}(t, 0) \zeta^{\infty}(0)
$$

in $C\left([0,1], H_{-\alpha}\right)$. Clearly, it suffices to show that

$$
\begin{gathered}
U^{\infty}(t, 0) \zeta^{N}(0) \Rightarrow U^{\infty}(t, 0) \zeta^{\infty}(0), \\
{\left[U^{\infty}(t, 0)-U^{N}(t, 0)\right] \zeta^{N}(0) \Rightarrow \delta_{0} .}
\end{gathered}
$$

The convergence of $(5.43)$ follows immediately from the convergence of $\zeta_{0}^{N}$ to $\xi_{0}^{\infty}$ since $U^{\infty}$ is just a "change of coordinate" in $C\left([0,1], H_{-\alpha}\right)$.

Let $V^{N}=U^{\infty}-U^{N}$. Then

$$
\left\|V^{N} \zeta^{N}\right\|_{-\alpha}=\sup _{\|J\|_{\alpha} \leqq 1}\left\langle J, V^{N} \zeta^{N}\right\rangle=\sup _{\|J\|_{\alpha} \leqq 1}\left\langle\left(V^{N}\right)^{*} J, \zeta^{N}\right\rangle \leqq \gamma_{N}\left\|\zeta^{N}\right\|_{-\alpha},
$$

where $\gamma_{N}$ is defined by

$$
\gamma_{N}=\sup _{\|J\|_{x} \leqq 1}\left\|\left(V^{N}\right)^{*} J\right\|_{\alpha}
$$

Clearly, $\gamma_{N} \rightarrow 0$ as $N \rightarrow \infty$. Thus $\left\|V^{N} \zeta^{N}\right\|_{-\alpha} \rightarrow 0$ with probability 1 and we conclude (5.44). Combining (5.41) to (5.44) we have proved Theorem 1.

\section{Appendix}

We shall prove Lemma 3.1 in this appendix. Let us first recall the following version of the asymptotic expansion of the central limit theorem [7].

Lemma A.1. Let $\left\{X_{j}\right\}$ be a sequence of i.i.d. with mean zero and variance $\sigma^{2}>0$. Let $f(x)$ be the common density. Suppose that $f^{\prime}(x)$ is integrable and $E\left[X_{1}^{8}\right]<\infty$. Let $g_{N}$ be the density of $\sigma^{-1} N^{-1 / 2} \sum_{j=1}^{N} X_{j}$. Then $g_{N}$ satisfies

$$
g_{N}(x)=(2 \pi)^{-1 / 2} \exp \left(-x^{2} / 2\right)\left[1+\sum_{\nu=1}^{6} N^{-v / 2} p_{v}(x)\right]+o\left(N^{-3}\right)
$$

uniformly in $x$. Here $p_{v}$ is a real polynomial of degree $3 v$ with coefficients depending only on $E X^{j} \equiv \mu_{j}, j=1, \ldots, 8$. For example,

$$
\begin{aligned}
& p_{1}(x)=\mu_{3} H_{3} / 6 \sigma^{3}, \\
& p_{2}(x)=\left[\mu_{3}^{2} H_{6} / 72 \sigma^{6}\right]+\left[\left(\mu_{4}-3 \sigma^{4}\right) H_{4} / 24 \sigma^{4}\right],
\end{aligned}
$$

with $H_{j}$ the Hermite polynomial of degree $j$ :

$$
\begin{aligned}
& H_{3}(x)=x^{3}-3 x, \quad H_{4}(x)=x^{4}-6 x^{3}+3, \\
& H_{6}(x)=x^{6}-15 x^{4}+45 x^{2}-15 .
\end{aligned}
$$

Proof of Lemma 3.1. We shall assume without loss of generality that $y=0$ and $\exp (-\phi(x))$ has mean 0 . By subtracting a constant from $F$ if necessary, we can 
assume that $\int F(x) \exp (-\varphi(x)) d x=0$. Let us choose a $\mu$ such that $(L \equiv 2 K+1)$

$$
\int x \exp \left(\mu x+\beta L^{-1} F(x)-\phi(x)\right) d x=0 .
$$

Clearly, $\mu$ can be solved as

$$
\mu=-\beta\langle x F(x)\rangle\left\langle x^{2}\right\rangle^{-1} L^{-1}+O\left(\beta^{2} / K^{2}\right),
$$

where $\langle>$ denotes expectation with respect to the density $\exp (-\phi(x))$. Let $Z$ be a random variable with density given by

$$
\exp \left(\mu x+\beta L^{-1} F(x)-\phi(x)\right) M(\mu, F)^{-1},
$$

where $M(\mu, F)$ is the normalization. Let $\left\{Z_{j}\right\}$ be the i.i.d. with density given by (A.3) and let $g_{Z, L}\left(\right.$ with $g_{L} \equiv g_{Z(\mu=0, \beta=0), L}$ ) denote the density of $L^{-1} \sigma^{-1} \sum_{i=1}^{L} Z_{i}$. Then
we can express the expectation in (3.5) as

$$
M(\mu, F)^{L} \sigma g_{Z, L}(0)\left[\sigma_{Z} g_{L}(0)\right]^{-1}, \quad \rho_{Z}^{2}=E\left[Z^{2}\right] .
$$

By (A.2) and Lemma A.1 we have

$$
\begin{aligned}
M(\mu, F) & =1+\beta^{2} G_{2}(F, 0) L^{-2}+O\left(\beta^{3} / K^{3}\right), \\
\sigma_{Z}^{2} & =M(\mu, F)^{-1} \sigma^{2}\left\{1-2 \beta G_{1}(F, 0)+O\left(\beta^{2} / K^{2}\right)\right\}, \\
g_{Z, L}(0) & =(2 \pi)^{-1 / 2}\left\{1+\sum_{v=2}^{6} L^{-v / 2} p_{Z, v}(0)\right\}+o\left(K^{-3}\right) .
\end{aligned}
$$

Here the expectation in $p_{Z, v}$ is with respect to the random variable $Z$. Since the difference between $p_{v}(0)(Z=X)$ and $p_{Z, v}(0)$ is of order $|\beta| K^{-1}$, we have

$$
\left|1-g_{Z, L}(0) / g_{L}(0)\right|<\text { const. } \beta / K^{2} \text {. }
$$

Lemma 3.1 follows from (A.4)-(A.8).

Acknowledgements. We wish to thank S. R. S. Varadhan for suggesting this problem and for his numerous stimulating discussions during this work. We also think P. Calderon for pointing out that the large field behavior in Theorem 2 can be estimated directly. This helps in simplifying the original proof. We also thank the refree for providing the last three references.

\section{References}

1. Brascamp, H. J., Lieb, E. H.: On extensions of the Brunn-Minkowski and Prékopa-Leindler theorems, including inequalities for $\log$ concave functions, and with an application to the diffusion equation, J. Funct. Anal. 22(4), 336-389 (1976)

2. Chang, C.-C.: Private communication

3. De Masi, A., Ianiro, N., Pellegrinotti, A., Presutti, E.: A survey of the hydrodynamical behavior of many-particle systems. In: Nonequilibrium phenomena II, Lebowitz, J. L., Montroll, E. W. (eds.) Amsterdam: North-Holland 1984

4. Fritz, J.: On the hydrodynamic limit of a scalar Ginzburg-Landau lattice model: The resolvent approach. In: Hydrodynamic Behavior and Interacting particle system, IMA volumes in Math. Appl. 9, G. Papanicolaou (ed.), pp. 75-97

5. Fritz, J.: On the hydrodynamic limit of a Ginzburg-Landau lattice model. Prob. Th. Rel. Fields 81, 291-318 (1989) 
6. Guo, M. Z., Papanicolaou, G. C., Varadhan, S. R. S.: Nonlinear diffusion limit for a system with nearest neighbor interactions. Commun. Math. Phys. 118, 31-59 (1988)

7. Petrov, V. V.: Sums of independent random variables. Berlin, Heidelberg, New York: Springer 1975

8 Rezakhanlou, F.: Hydrodynamic limit for a system with finite range interactions. Commun. Math. Phys. 129, 445-480 (1990)

9. Richtmyer, R. D., Morton, K. W.: Difference methods for initial-value problems, 2nd ed. New York: John Wiley 1967

10. Spohn, H.: Equilibrium fluctuations for interacting Brownian particles. Commun. Math. Phys. 103, 1-33 (1986)

11. Spohn, H.: Large scale dynamics of interacting particles, Part B. Stochastic lattice gases, preprint, 1989

12. Stroock, D. W., Varadhan, S. R. S.: Multidimensional Diffusion Processes. Berlin, Heidelberg, New York: Springer 1979

13. Varadhan, S. R. S.: Stochastic Processes. Courant Inst. Lecture Notes

14. Varadhan, S. R. S.: Large Deviations and Applications, CBMS-NSF Regional Conference Series in Applied Mathematics, Vol. 46, SIAM (1984)

15. Zhu, Ming: Equilibrium fluctuations for one-dimensional Ginzburg-Landau lattice model. Nagoya Math. J. 17, 63-92 (1990)

16. Deuschel, J., Stroock, D.: Large Deviations. New York: Academic Press 1989

17. Dittrich, P.: A stochastic particle system: Fluctuations around a nonlinear reaction-diffusion equation. Stochastic Processes Appl. 30, 149-164 (1988)

18. Ferrari, P. A., Presutti, E., Vares, M. E.: Nonequilibrium fluctuations for a zero range process, V24, No. 2, 237-268 (1988)

19. Spohn, H.: Equilibrium fluctuations for some stochastic particle systems in statistical physics and dynamical systems, J. Fritz, A. Jaffee, D. Szász (eds.) Boston: Birkhäuser 1985

Communicated by J. L. Lebowitz 